\title{
The genome and developmental transcriptome of the strongylid nematode Haemonchus contortus
}

\author{
Erich M Schwarz ${ }^{1,2,3 \dagger}$, Pasi K Korhonen ${ }^{2 \dagger}$, Bronwyn E Campbell ${ }^{2 \dagger}$, Neil D Young ${ }^{2 \dagger}$, Aaron R Jex ${ }^{2}$, Abdul Jabbar ${ }^{2}$, \\ Ross S Hall ${ }^{2}$, Alinda Mondal ${ }^{2}$, Adina C Howe ${ }^{4}$, Jason Pell ${ }^{5}$, Andreas Hofmann 6 , Peter R Boag ${ }^{7}$, Xing-Quan Zhu ${ }^{8}$, \\ T Ryan Gregory ${ }^{9}$, Alex Loukas ${ }^{10}$, Brian A Williams ${ }^{1}$, Igor Antoshechkin ${ }^{1}$, C Titus Brown $^{4,5}$, Paul W Sternberg ${ }^{1}$ and \\ Robin B Gasser ${ }^{2^{*}}$
}

\begin{abstract}
Background: The barber's pole worm, Haemonchus contortus, is one of the most economically important parasites of small ruminants worldwide. Although this parasite can be controlled using anthelmintic drugs, resistance against most drugs in common use has become a widespread problem. We provide a draft of the genome and the transcriptomes of all key developmental stages of $\mathrm{H}$. contortus to support biological and biotechnological research areas of this and related parasites.

Results: The draft genome of $\mathrm{H}$. contortus is $320 \mathrm{Mb}$ in size and encodes 23,610 protein-coding genes. On a fundamental level, we elucidate transcriptional alterations taking place throughout the life cycle, characterize the parasite's gene silencing machinery, and explore molecules involved in development, reproduction, host-parasite interactions, immunity, and disease. The secretome of $\mathrm{H}$. contortus is particularly rich in peptidases linked to bloodfeeding activity and interactions with host tissues, and a diverse array of molecules is involved in complex immune responses. On an applied level, we predict drug targets and identify vaccine molecules.

Conclusions: The draft genome and developmental transcriptome of $H$. contortus provide a major resource to the scientific community for a wide range of genomic, genetic, proteomic, metabolomic, evolutionary, biological, ecological, and epidemiological investigations, and a solid foundation for biotechnological outcomes, including new anthelmintics, vaccines and diagnostic tests. This first draft genome of any strongylid nematode paves the way for a rapid acceleration in our understanding of a wide range of socioeconomically important parasites of one of the largest nematode orders.
\end{abstract}

\section{Background}

The strongylid nematode Haemonchus contortus (barber's pole worm) is one of the most important parasites of livestock, and represents a large order of nematodes (Strongylida) that infect both animals and humans worldwide [1-3]. H. contortus infects hundreds of millions of sheep and goats globally, and causes deaths and production losses estimated at tens of billions of dollars per annum. This nematode feeds on blood from capillaries in the stomach mucosa, and causes hemorrhagic gastritis, anemia, edema and associated complications, often leading to

\footnotetext{
* Correspondence: robinbg@unimelb.edu.au

† Contributed equally

${ }^{2}$ Faculty of Veterinary Science, The University of Melbourne, Corner of

Flemington Road and Park Drive, Parkville, Victoria 3010, Australia

Full list of author information is available at the end of the article
}

death of severely affected animals [2]. H. contortus is transmitted orally from contaminated pasture to the host through a complex 3-week life cycle [4]: the eggs are excreted in the host feces, the first-stage larva (L1) develops inside the egg, then hatches (within about 1 day) and molts through to the second-stage (L2) and third-stage (L3) larval stages within approximately 1 week; the infective L3s are then ingested by the host, exsheath, and, after a histotropic phase, develop through the fourth-stage larvae (L4s) to dioecious adults; these two last stages both feed on blood.

Only four main drug classes have been available for the treatment of strongylid infections, and resistance against these classes is spreading worldwide [5]. It is thus highly desirable to search for new drug targets encoded in the $H$. contortus genome. Although vaccines

\section{Biomed Central}


using some native parasite antigens (called H11 or H-gal-GP) can partially prevent haemonchosis in experimental sheep, homologous recombinant molecules have failed to achieve protection [6]. Therefore, current treatment relies predominantly on the use of nematocidal drugs (anthelmintics). Because resistance against the main classes of drugs has become widespread [5], the ongoing design of new compounds, such as monepantel [7], cyclooctadepsipeptides [8], and derquantel-abamectin [9], is required. Discovering new drugs has been challenging, particularly because of the current limited understanding of the biology of $H$. contortus and how it interacts with its host [2]. Here, we describe a draft genome and developmentally staged transcriptome of $H$. contortus to substantially improve our understanding of this parasite at the molecular level. This genome provides a major resource to the scientific community for a wide range of genomic, genetic, evolutionary, biological, ecological, and epidemiological investigations, and a solid foundation for the development of new interventions (drugs, vaccines and diagnostic tests) against $H$. contortus and related strongylid nematodes.

\section{Results and discussion}

\section{Sequencing and assembly}

We sequenced the genome of $H$. contortus (McMaster strain, Australia) at 185 -fold coverage (Table 1; see Additional file 1 Table S1), producing a final draft assembly of $320 \mathrm{Mb}$ (scaffold N50: $56.3 \mathrm{~kb}$; Table 1) with a mean GC content of $42.4 \%$. We detected $91.5 \%$ of 248 core essential genes by CEGMA, suggesting that the assembly represents a substantial proportion of the entire genome. The estimated repeat content for this draft genome is $13.4 \%$, equating to $42.8 \mathrm{Mb}$ DNA. To overcome challenges in the assembly of the genome, we removed excessive repetitive and erroneous reads by khmer filtering [10] and normalization [11] to produce a representative assembly, an approach that should be useful for other complex genomes. This assembly contained $2.0 \%$ retrotransposons and 2.1\% DNA transposons, which is similar to that reported for some other nematode genomes sequenced to date, including those of Caenorhabditis elegans, Pristionchus pacificus, and Ascaris suum [12-14]. We identified 40,046 retrotransposon sequences (see Additional file 1, Table S2) representing at least 9 families (4 long terminal repeats (LTRs), 3 long interspersed nuclear elements (LINEs), and 2 short interspersed nuclear elements (SINEs)). We also identified two families of DNA transposons (36,861 distinct sequences in total) and 235,635 unclassified repeat elements. The most abundantly transcribed repeat elements were DNA/TcMar and LINEs/retrotransposable elements (RTEs; see Additional file 1, Table S2). This richness of transposable element families is substantially higher than that predicted for other genomes of parasitic nematodes [13-16]. Overall, the present draft genome $(320 \mathrm{Mb})$ is the largest of any animal parasitic nematode sequenced to date (for example, 273, 89 , and $64 \mathrm{Mb}$ for A. suum, Brugia malayi, and Trichinella spiralis, respectively)[14-16].

\section{H. contortus gene set}

Using transcriptomic data from egg, larval, and adult stages of $H$. contortus (Haecon-5 strain, Australia), de novo predictions and homology-based searching, we annotated 23,610 genes, all of which are supported by transcriptomic and protein data, with a mean total length of 6,167 bp, exon length of $139 \mathrm{bp}$, and estimated 7.2 exons per gene (Table 1). Mean gene and intron lengths $(6,167$ and $832 \mathrm{bp})$ for $H$. contortus were comparable

Table 1 Features of the Haemonchus contortus draft genome

\begin{tabular}{ll}
\hline Description & \\
\hline Total number of base pairs within assembled scaffolds & $319,640,208$ \\
Total number of scaffolds; contigs & 14,$419 ; 930,981$ \\
N50 length in bp; total number > 2 kb in length & 56,$328 ; 11,000$ \\
N90 length in bp; total number > N90 length & 13,$105 ; 6,085$ \\
GC content of the whole genome (\%) & 42.4 \\
Repetitive sequences (\%) & 13.4 \\
Proportion of genome that is coding (exonic; incl. introns) (\%) & $8.6 ; 43.3$ \\
Number of putative coding genes & 23,610 \\
Gene size (mean bp) & 6,167 \\
Average coding domain length (mean bp) & 835 \\
Average exon number per gene (mean) & 7 \\
Gene exon length (mean bp) & 139 \\
Gene intron length (mean bp) & 832 \\
GC content in coding regions (\%) & 45.4 \\
Number of transfer RNAs & 449 \\
\hline
\end{tabular}


with those of $A$. suum (6,536 and 1,081 bp), but greater than those for other nematodes, such as C. elegans, B. malayi, and T. spiralis (around 1,000 to 2,000 and around 100 to $400 \mathrm{bp}$ ). Most of the predicted $H$. contortus genes (Figure 1) were found to have homologs (BLASTp e-value cut-off $10^{-5}$ ) in other nematodes (16,545; 70.0\%), including C. elegans $(15,907 ; 67.4 \%)$, A. suum $(14,065 ; 59.6 \%)$, B. malayi $(12,129 ; 51.4 \%)$, and T. spiralis $(9,326 ; 39.5 \%)$. In total, 8,505 genes were found to be orthologous among the five species, with 608 being shared with at least one other species of nematode but absent from C. elegans (Figure 1). Conversely, 7,095 genes $(30.1 \%)$ were found to be unique to $H$. contortus relative to the other four species (Figure 1). Conspicuous were at least 325 genes that are exclusive to all four parasitic nematodes and that are included here for comparison $\left(\leq 10^{-5}\right)$. Of the entire $H$. contortus gene set, 5,213 genes had an ortholog $\left(\leq 10^{-5}\right)$ linked to one of 291 known biological pathways (Kyoto Encyclopedia of Genes and Genomes; KEGG; see Additional file 1, Table S3). Mapping to pathways in C. elegans suggested a near-complete complement of genes, also supporting the CEGMA results. By inference, essentially all of the $H$. contortus genes are represented in the present genomic assembly, and are supported by extensive transcriptomic and inferred protein data. Using data for C. elegans and data available in all accessible protein- and/or conserved protein domain-databases, we predicted functions (including enzymes, receptors, channels, and transporters) for
19,391 (77.92\%) of the protein-coding genes of $H$. contortus (Table 2).

We identified 429 peptidases representing five key classes (aspartic, cysteine, serine, and threonine peptidases and metallopeptidases), with the metallopeptidases $(n=141 ; 32.9 \%)$ and serine peptidases $(107 ; 24.9 \%)$ predominating (see Additional file 1, Table S4). Notable were secreted peptidases, such as astacins (M12A), neprilysins (M13), selected serine peptidases (SC; S09), cathepsins (C01A), and calpain-2s (C19), which are abundantly represented and, based on information available for other nematode species [13-15,17], likely to have key roles in host invasion, locomotion, migration into stomach tissue (during the histotropic phase), degradation of blood and other proteins, immune evasion, and/or activation of inflammation. We also identified 845 kinases and 330 phosphatases in $H$. contortus (see Additional file 1, Tables S5 and S6). All major classes of kinases are represented, with tyrosine kinase $(n=92)$, casein kinase $1(n=90)$, CMGC $(n=67)$, and calcium/calmodulin-dependent protein kinase $(n=$ 65 ) homologs being abundant (37.2\%), and a similar number of unclassified kinases (37\%). The phosphatome includes mainly protein tyrosine $(n=69)$, serine/threonine $(n=50)$, receptor type tyrosine $(n=32)$, histidine $(n=31)$, and dual-specificity $(n=25)$ phosphatases. Based on homology with $C$. elegans proteins, we predicted 247 GTPases, including 215 small GTPases representing the Rho $(n=50)$, Rab $(n=38), \operatorname{Ran}(n=57)$, Arf

\section{H. contortus}

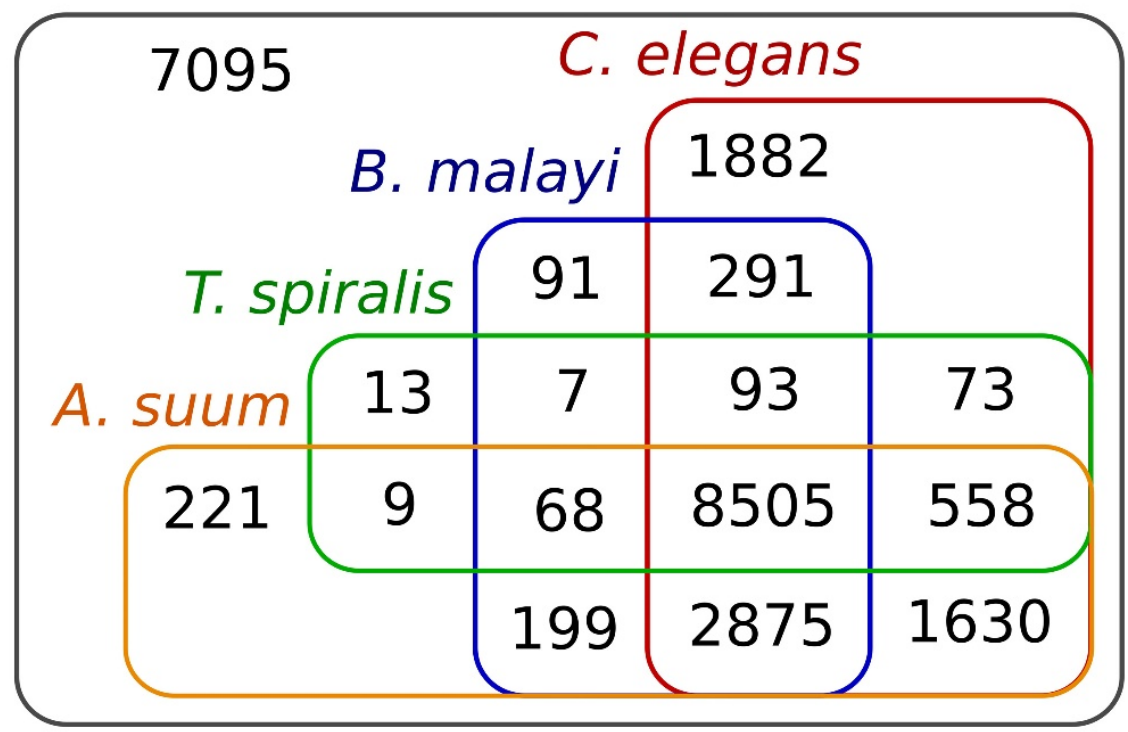

Figure 1 Venn diagram showing the numbers of homologs between Haemonchus contortus and four other nematode species (Ascaris suum, Brugia malayi, Caenorhabditis elegans, and Trichinella spiralis) after pairwise comparison. 


\begin{tabular}{ll}
$\begin{array}{l}\text { Table } 2 \text { Major protein groups representing the } \\
\text { Haemonchus contortus gene set }\end{array}$ \\
\hline Protein group $^{\text {a }}$ & $\begin{array}{l}\text { Number } \\
\text { predicted }\end{array}$ \\
\hline Channels & 2,454 \\
Ligand-gated ion channels (LGICs) & 297 \\
G protein-coupled receptors (GPCRs) & 540 \\
GTPases & 247 \\
Major sperm proteins (MSPS) & 42 \\
Vitellogenins & 3 \\
Peptidases & 429 \\
Peptidase inhibitors & 119 \\
Kinases & 845 \\
Phosphatases & 330 \\
RNAi machinery & 229 \\
Secretome & 1,457 \\
SCP/TAPS & 84 \\
Structural proteins & 943 \\
Other proteins with known homologues and/or & 11,710 \\
domains & \\
Hypothetical proteins & 5,378 \\
\hline
\end{tabular}

${ }^{\mathrm{a}}$ Some predicted proteins belonged to multiple categories.

$(n=23)$, and Miro $(n=2)$ families, and a small number of large GTPases (such as dyamin, GBP, and mitofusin; $n=15$; see Additional file 1, Table S7). Examples of small

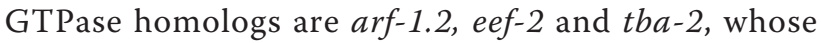
C. elegans orthologs are essential for embryonic, larval, and/or reproductive development. Therefore, some of these enzymes were proposed as targets for anti-parasite interventions $[18,19]$. Similarly, the large range of channel, pore, and transporter proteins that we identified here is of particular interest in this context, considering that many common anthelmintics bind representatives of some of these proteins as targets [7]. For H. contortus, we predicted $540 \mathrm{G}$ protein-coupled receptors (GPCRs), most of which belonged to classes SR $(n=299)$ and A (147; see Additional file 1, Table S8). In addition, we identified 786 channel or pore proteins, including voltage-gated ion channels (VICs) and ligand-gated ion channels (LGICs; see Additional file 1, Table S9). Such channels are known targets for nematocidal drugs, such as macrocyclic lactones (for example, cydectin), levamisole and monepantel (an aminoacetonitrile derivative; AAD) [7]. Importantly, in the H. contortus gene set, we found a homolog acr-23 of the C. elegans monepantel receptor, supporting evidence that this drug kills $H$. contortus in vivo [7]. In addition, we detected an abundance of transporters, including 617 electrochemical potential-driven (almost all porters) and 526 primary active (mainly P-Pbond-hydrolysis-driven) transporters, and 308 transportassociated molecules (see Additional file 1, Table S9).

Excretory/secretory (ES) proteins are central to the parasite-host relationship [19]. We predicted the secretome of
$H$. contortus to comprise 1,457 proteins with a diverse range of functions (see Additional file 1, Table S10). Most notable were 318 peptidases, including 98 metallopeptidases and 68 cysteine, 67 aspartic, 19 serine peptidases (predominantly clans MA, CA, AA, and SA, respectively) and 66 peptidase inhibitors (including fibronectin type III), 90 lectins (including C-type and concanavalin A-like), 65 sperm-coating protein/Tpx-1/Ag5/PR-1/Sc7 (SCP/TAPS) proteins, 38 transthyretin-like (TTL) proteins, and 27 kinases. Many secreted peptidases (comprising the 'degradome') and their respective inhibitors have known roles in the penetration of tissue barriers and feeding for a range of parasitic worms, including $H$. contortus $[2,6,18]$. Some of these ES proteins are involved in host interactions and/ or inducing or modulating host immune responses against parasitic worms, which are often Th2-biased [19].

\section{Key transcriptional changes during developmental transitions in the life cycle}

$H$. contortus development involves a number of tightly timed processes [4]. Embryogenesis generates the basic tissue types of the nematode, and each tissue type differentiates at a specific point in the developmental cycle. Post-embryonic structures required for parasitism and reproduction then differentiate in the larval stages $\mathrm{L} 1$ to L4. This includes the specialized development of the buccal capsule for blood feeding (from L4 onward), sexual differentiation at the L4 stage, and gametogenesis in the adult stage. Substantial growth occurs at the L2, L4, and adult stages. Development occurs in two different environments, on pasture for the free-living stages L1 to L3, and in the host for the dioecious L4 and adult stages (Figure 2). Each of these stages has different requirements, in terms of motility, sensory perception, metabolism, and the regulation of hormones of the endocrine system. L3, which is the infective stage, and thus represents the transitional stage from a free-living to parasitic organism, persists in the environment until it is ingested by the host, where it then receives a signal (mainly $\left.\mathrm{CO}_{2}\right)$ to commence its development as a parasite. The complexity of the $H$. contortus life cycle coincides with key developmental alterations in the nematode that probably require tightly controlled and rapidly regulated transcriptional changes.

We studied differential transcription from stage to stage, as the parasite developed from egg to adult (Figure 2). The transition from the undeveloped egg to L1 was associated with significant upregulation of transcription for 1,621 genes encoding a substantial number of channels $(n=641)$, including LGICs and ES proteins $(n=397)$, GPCRs (134), transcription factors (TFs; $n=$ $43)$, kinases $(n=100)$, and phosphatases $(n=35$; see Additional file 1, Table S11). Although this expansion is probably associated with mitosis, organelle biogenesis, 


\section{Major upregulation in blood-feeding stages:}

Structural molecules (collagens); ES proteins (SCP/TAPS proteins); peptidases for tissue degradation and feeding; growth, development and reproduction; respiratory electron transport chain; haemoblobin-like proteins involved in oxygen uptake, osmotic regulation, iron storage or oxygen-detoxification; enzymes linked to haeme transport or detoxification of reactive oxygen species.

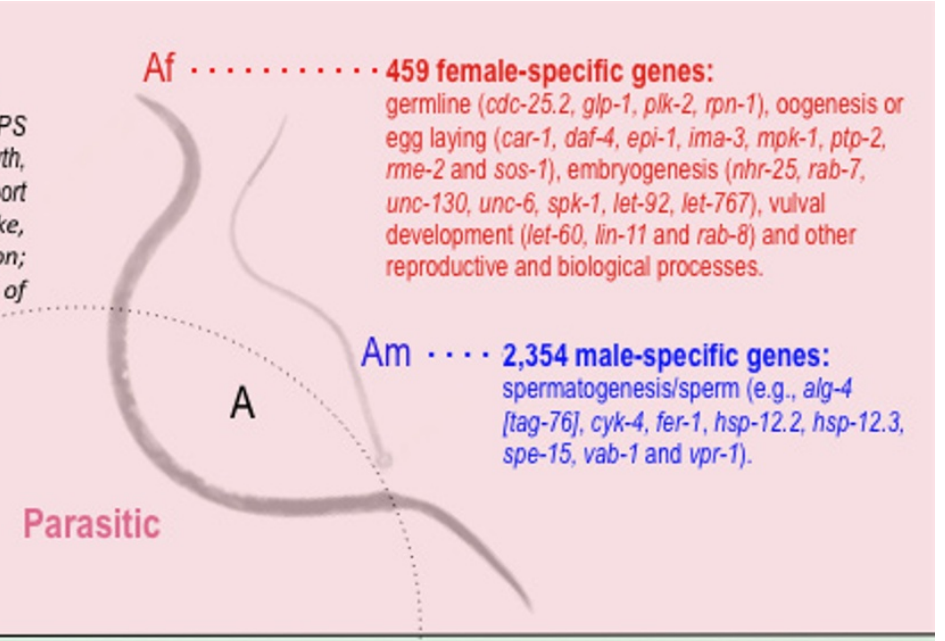

L4

\section{(n)

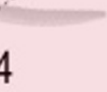

A spermatogenesis/sperm (e.g., alg-4 [tag-76], cyk-4, fer-1, hsp-12.2, hsp-12.3, spe-15, vab-1 and vpr-1).

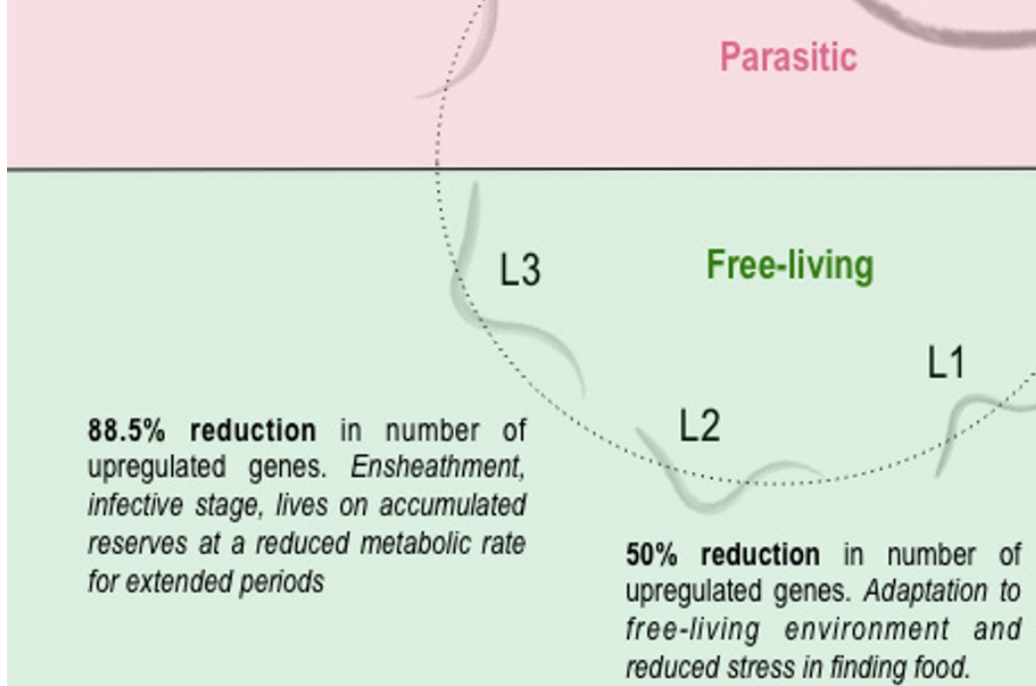

E

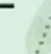

Upregulation of 1,621 genes (100\%) encoding channels, ES proteins, GPCRs, transcription factors, kinases and phosphatases. Mitosis, organelle biogenesis, apoptosis and overall gene expression; chemo-, osmo- and touch-sensation, proprioception; search for microbial food sources.

Figure 2 Transcriptional changes in the life cycle of Haemonchus contortus. In a 3-week life cycle of the parasite, eggs (E) are excreted in host feces; the first-stage larva (L1) develops inside the egg to hatch and molt through to the second-stage (L2) and third-stage (L3) larval stages within a week. The infective L3s are then ingested by the small ruminant host, where they exsheath and, after a short tissue phase, develop through the fourth-stage larval (L4) stage to dioecious adults; both of these stages feed on host blood from capillaries in the internal wall of the stomach. Disease in the host relates to this blood-feeding activity. In this figure, changes in transcription in the transition from stage to stage are summarized and interpreted in the context of the biology of the parasite. Information is given on key genes differentially transcribed between adult female (Af) and male (Am) H. contortus, and involved in reproductive and other biological processes; gene codes follow those of Caenorhabditis elegans orthologs.

apoptosis, and overall gene expression during the rapid growth and development of L1 [4], based on knowledge of C. elegans [20], the expansion of some key subsets of channels, pore and electrochemical potential-driven transporters, GPCRs (classes A and SR), and various kinases/ phosphatases probably relate to chemosensation, mechanosensation, osmosensation, and/or proprioception of the free-living L1, as it rapidly adapts to its new and harsh external environment.

The activity of L1s of $H$. contortus and their search for microbial food sources might reflect the expansion of ES proteins and associated peptidases and their inhibitors (Figure 2). The switch to L2 sees an approximately $50 \%$ reduction in number of upregulated genes of the same groups, possibly reflecting the gradual adaptation to its free-living environment and a reduced level of stress in finding food. The transition to the L3 stage sees an $88.5 \%$ reduction in the number of differentially transcribed genes representing the same groups (Figure 2), an expected finding, given that this stage undergoes ensheathment, is no longer able to feed, and must live on accumulated reserves at a reduced metabolic rate in order to survive (as an 'arrested' but motile infective L3 stage) for extended periods in the external environment [2]. Once ingested by the host animal, the transition from the L3 to the parasitic L4 and adult stages sees a renewed, massive surge in the number of differentially transcribed genes of the same spectrum of molecules and of structural proteins, but, as expected, very limited differences between the L4 and adult stages, with the exception of some genes 
(for example, those encoding vitellogenin) that appear to relate specifically to reproduction (Figure 2; see Additional file 1, Table S11).

During the key transitions in the life cycle (from egg to L2, and then from L3 to L4) linked to substantial growth and development [2], a range of genes encoding collagens and cuticular proteins are upregulated per transition (Figure 2; see Additional file 1, Table S11). In the nematode cuticle, such molecules are crucial for the maintenance of nematode body shape, and also for protection against and contact with the external environment or host interface. We found prominent variation in transcription profiles among 28 individual collagen genes in the transition from the free-living to parasitic stages, consistent with previous immunoproteomic findings [21].

More than 120 peptidase genes were significantly upregulated in blood-feeding stages (Figure 2; see Additional file 1, Table S11). Conspicuous among them were genes encoding secreted peptidases of various clans, including MA (metallopeptidases; M12A, M01, M13, M12A, M10A), AA (aspartic peptidases; all A01A) and CA (cysteine peptidases; mostly CA01A; see Additional file 1, Table S11), which have known roles in the degradation of tissues during the parasite's histotropic phase and digestion of blood components following establishment and buccal-capsule attachment to the abomasal wall, and might be crucial for growth, development, and survival of $H$. contortus in the host animal [2]. These findings support previous evidence showing that, for example, cysteine peptidases play a crucial role in the catabolism of globin by the cleavage of hemoglobin in blood-feeding nematodes [22-25]. Concomitantly, in the blood-feeding stages, we observed upregulated transcription of genes encoding succinate dehydrogenase subunit B and glutamate dehydrogenase genes via the respiratory electron transport chain [26] (proposed to maintain the redox balance in response to the accumulation of the end products from anaerobic metabolism [27]), and hemoglobin-like proteins [28] (probably involved in oxygen uptake, osmotic regulation, iron storage and/or oxygen-detoxification [29]). We also found increased transcription of genes encoding enzymes, including glutathione $\mathrm{S}$-transferase, cytoplasmic $\mathrm{Cu} / \mathrm{Zn}$ superoxide dismutase, catalase, glutathione peroxidase and/or peroxiredoxin, which are likely to have roles in heme transport or detoxification of reactive oxygen species from endogenous metabolic activities from the host during $H$. contortus infection; this is supported by findings from previous investigations [30-32] and recognized as characteristic of tissue-dwelling or blood-dwelling parasites [32].

The initiation of reproduction in adult $H$. contortus was marked by a developmentally regulated transcription of sex-enriched genes. Using a networking approach $[33,34]$, we identified clusters of genes whose transcripts are significantly differentially transcribed (four-fold) between female and male adults of $H$. contortus. The totals of 459 female-specific and 2,354 male-specific genes represent 397 (degree: 10) and 1,620 (degree: 10) cluster hubs, respectively (Figure 2; see Additional file 1, Table S12). We found that both female and male gene sets were enriched for genes associated with growth, genital, embryonic, and germline development, and reproduction. Within the female set were genes associated with germline (for example, $c d c-25.2, g l p-1, p l k-2$, and rpn-1), oogenesis or egg laying (for example, car-1, daf-4, epi-1, ima-3, mpk-1, ptp-2, rme-2, and sos-1), embryogenesis (for example, $n h r-25, r a b-7, u n c-130, u n c-6, s p k-1$, let-92, and let-767), vulval development (for example, let60 , lin-11, and $r a b-8$ ), and other reproductive and biological processes. Notable within the male set were genes associated specifically with spermatogenesis/sperm (for example, alg-4 [tag-76], cyk-4, fer-1, hsp-12.2, hsp-12.3, spe-15, vab-1, and vpr-1). There are at least 977 sexenriched genes $(34.7 \%)$ in $H$. contortus that do not have homologs in other organisms.

\section{Parasite-host interactions}

Considering the substantial attack against $H$. contortus within the host, many ES proteins are expected to play crucial roles during parasite establishment, infection, immune modulation, or evasion. This expectation is supported by abundant transcription in the L4 and adult stages of genes encoding peptidases $(n=142)$, SCP-like extracellular proteins (including 20 neutrophil inhibitory factors; NIFs), lectins $(n=23)$, TTL proteins $(n=10)$, peptidase inhibitors $(n=6$; including 4 Kunitz-like molecules) and fatty acid retinoid binding proteins $(n=$ 4; see Additional file 1, Tables S10 and S13). In total, 333 of 1,457 genes (22.9\%) encoding ES proteins were transcribed at significantly higher levels in the parasitic compared with the free-living stages (see Additional file 1, Table S10). The genome-wide average for this upregulation was significantly lower $\left(14.7 \% ; P=5.8 \times 10^{-12}\right)$.

In the hematophagous stages, we identified 54 upregulated genes encoding SCP/TAPS proteins [35], characterized by one or more SCP-like extracellular domains (IPR014044 and/or IPR001283). These proteins, originally found in hookworms, are also called activation-associated proteins or Ancylostoma-secreted proteins (ASPs) [36,37]. Although the numbers of genes inferred to express SCP/ TAPS proteins were similar between the L4 and adult stages (see Additional file 1, Tables S11 and S14), there were qualitative and quantitative differences in transcription compared with other developmental stages. Although two genes encoding the SCP/TAPS proteins Hc24 and Hc40 were identified previously in ES products of adult $H$. contortus [38,39], we identified 82 more such genes (see Additional file 1, Table S14). This finding supports a 
previous proposal for a wide array of molecules of this group in $H$. contortus [40], and suggests a diversified, active, and specific involvement of SCP/TAPS proteins in infection. Of the $84 \mathrm{SCP} / \mathrm{TAPS}$ proteins (62 single and 22 double SCP-like domain molecules) encoded by $H$. contortus, 74 were found to have homologs in C. elegans (see Additional file 1, Table S14); the $10 \mathrm{H}$. contortus-unique SCP/TAPS proteins, some of which are upregulated in parasitic stages, probably relate to host interactions and/or disease. Although SCP/TAPS proteins are still enigmatic, in terms of their functions, they deserve detailed investigation, particularly given that they are being explored as vaccine candidates for other hematophagous nematodes. For instance, in the human hookworm Necator americanus, $\mathrm{Na}$-ASP-2 was tested in a phase I clinical trial, owing to its known protective properties in humans [41], although initial vaccination with the recombinant protein in adjuvant resulted in unexpected allergic responses following natural exposure to the parasite [42]. The crystal structure of Na-ASP-2 shows charge segregation similar to that of mammalian chemokines, indicating that this molecule might be an agonist or ligand for some GPCRs, such as chemokine receptors [43]. Of the 84 SCP/TAPS proteins identified in $H$. contortus, 20 were NIFs and predicted to be abundant in ES products, and have already been found in some other nematodes [19]. Although NIFs have not been reported previously for $H$. contortus, an Ancylostoma caninum homolog (SCP-1) is known to bind host integrin CR3 (CD11b/CD18) and to be able to inhibit neutrophil function, including oxidative burst $[44,45]$.

As expected from previous molecular studies $[40,46]$, eight genes encoding NIM-like proteins were found to be abundant in the hematophagous stages of $H$. contortus (see Additional file 1, Table S13). Although the functional roles of NIM proteins are unclear, they are likely to be involved in host-parasite interactions, because they are abundantly transcribed in parasitic stages. Most have $\mathrm{N}$-terminal signal peptides and, thus appear to be actively excreted/secreted [40], although there is variation in the abundance of these proteins among different populations of $H$. contortus $[40,46,47]$.

Of 53 genes encoding TTL proteins, 10 were significantly upregulated in parasitic stages of $H$. contortus (see Additional file 1, Table S13). Most TTL proteins identified to date are relatively conserved across large evolutionary distances [48], and are enzymes of purine catabolism that catalyze the conversion of 5-hydroxyisourate to 2-oxo-4hydroxy-4-carboxy-5-ureidoimidazoline $[49,50]$. In metazoans, TTLs can also bind hormones, such as thyroxine (T4) and vitamin A [51], and can enable cell corpse engulfment by binding surface-exposed phosphatidylserine on apoptotic cells [52]. Among the proteins encoded by nematode-specific genes, TTLs represent one of the largest groups [53,54]. A subset of TTL proteins has also been identified in Ostertagia ostertagi, a nematode related to $H$. contortus $[55,56]$, in the human filarial nematode B. malayi [57], and in the plant-parasitic nematodes Xiphinema index, Heterodera glycines, Meloidogyne incognita, and Radopholus similis [53,58-60]. For example, in $O$. ostertagi, at least $18 \mathrm{ttl}$ genes have been identified by data mining, most of them being constitutively transcribed from the free-living L3 through to adult females and males [56]. In H. contortus, a TTL has been isolated from ES products from adult worms and shown to be immunogenic [40], and TTL homologs are also abundant in An. caninum ES [61]. These data suggest the testable hypothesis that TTLs, together with SCP/TAPS proteins, play key roles in host interactions.

\section{Immune responses}

Based on the current knowledge and understanding of immune responses against helminths in animals [19], we compiled a comprehensive list of $H$. contortus ES homologs with known immunomodulatory or immunogenic roles in other nematodes (see Additional file 1: Table S13). Such homologs upregulated in the L4 and adult stages represent $5.6 \%$ of the predicted $H$. contortus secretome, which is significantly lower than the genome-wide average of $14.7 \%\left(P<10^{-6}\right)$. In addition to the molecules HcES15 and HcES24, whose precise functions are still unclear, proteins within this secretome that are predicted to direct or suppress immune responses include close homologs of $\mathrm{N}$-acteylglycosaminyltransferase and leucyl aminopeptidase ES-62 of the filarioid nematode Acanthocheilonema vitae [19]. ES-62 is known to inhibit B-cell, T-cell and mast cell proliferation/responses, induce a Th2 response through the inhibition of IL-12p70 production by dendritic cells, and promote alternative activation of the host macrophages via the inhibition of Toll-like receptor (TLR) signaling [19]. Other molecules of $H$. contortus predicted to be immunomodulatory include homologs of another B-cell inhibitor (CYS-1), 8 serpins and 20 NIFs [19]. Some $H$. contortus ES proteins are predicted to be involved in immune evasion; for instance, some could mask parasite antigens by mimicking host molecules (for example, C-type lectins, concanavalin A and galectins) [19]. In spite of some similarities among nematode-host systems, based on the nature and extent of molecules identified, the host immune responses against the parasitic stages of $H$. contortus appear to be distinct from those associated with other nematodes, such as Ascaris and filarioids, which is supported by other experimental findings [19]. Taken together, the present findings indicate that $H$. contortus has a substantial arsenal of ES proteins that are likely to be involved in modulating, evading, and/or blocking immune responses in the host. 


\section{Vaccine molecules}

There has been a major emphasis on the development of vaccines to fight against haemonchosis [6]. Most effort has been directed at inducing immunity in sheep against proteins expressed in or excreted/secreted from the gut of $H$. contortus, with the aim of disrupting or inhibiting the parasite's digestion of host blood. To date, the two most effective immunogens assessed have been the aminopeptidase family $\mathrm{H} 11 \quad[62,63]$ and the Haemonchus galactose-containing glycoprotein complex (H-gal-GP) [64]. Both of these molecular complexes contain integral membrane proteins with hemoglobinase activity, are expressed mainly in the microvillar surface of the parasite's gut, and induce 70 to $90 \%$ protection against infection in a number of sheep breeds [6]. In the current study, using genomic and transcriptomic data, we were able to define the different molecular variants within these two complexes.

We found that H11 represents a group of 25 different metallopeptidases (clan MA; family M01; see Additional file 1, Table S15), which are upregulated six-fold to 210 -fold in the parasitic over the free-living stages of $H$. contortus. Key components of H-gal-GP, representing predominantly metallopeptidases (for example, MEPs 1 to 4) $[65,66]$, aspartyl peptidases (for example, HcPEPs 1 and 2) $[67,68]$, and cysteine peptidases (for example, AC-1 to AC-5; HMCP-1 to HMCP-6) [22,67,69-71], were also identified using sequence data from previous proteomic studies (see Additional file 1: Table S15). Again, as expected from previous studies [6], all three classes of peptidases were significantly upregulated in the L4 and adult stages (see Additional file 1, Table S15). We found substantial diversity in the cysteine peptidases $(n=$ 81 ), which have been also under close scrutiny as vaccine candidates. Many of these enzymes $(n=14)$ represent clan C01A (cathepsin B-like peptidases), and 34.6\% were represented in the ES degradome (see Additional file 1, Tables S4 and S16). We also identified 11 legumains (clan CD; family C13), which might activate key family C01A peptidases through cleavage of the peptide backbone between the pro-segment and mature enzyme domains [72].

In addition, the serine peptidase complex contortin has received attention as an efficient anticoagulant (with dipeptidyl IV activity) in parasitic stages of $H$. contortus [73]. Contortin is inferred to belong to clan SC serine peptidases (family S28). We found 13 family S28 representatives among the 107 serine peptidases predicted for H. contortus (see Additional file 1, Table S15), all of which were upregulated in the parasitic stages. Nine of these thirteen lysosomal Pro-Xaa carboxypeptidases were represented in the ES degradome (see Additional file 1, Table S16), supporting the contention that contortin is also immobilized [73]. Interestingly, $H$. contortus shares many of these key classes of peptidases with other hematophagous parasites, including hookworms, indicating relative conservation in sequence and function linked mainly to feeding (blood meal digestion or anticoagulation). Studies to date have shown that selected recombinant proteins representing $\mathrm{H} 11$ and $\mathrm{H}$-gal-GP do not induce protective immune responses, and carbohydrate moieties alone are also not protective [6]. Therefore, the combined use of proteomic and glycomic tools, underpinned by the present genomic and transcriptomic data sets as well as by animal experimentation, should be advantageous for designing future vaccines.

\section{Prediction and prioritization of drug targets}

The excessive and uncontrolled use of a small number of drug classes for the treatment of haemonchosis has led to major problems of drug resistance in $H$. contortus to most of these compounds [5]. Unfortunately, only a very small number of new anthelmintics (cyclooctodepsipeptides and aminoacetylnitriles) have been discovered in the past two decades using traditional chemical screening approaches [7,74]. Genome-guided drug target or drug discovery provides an alternative means to conventional screening and repurposing [75]. The aim of genome-guided discovery is to identify genes or molecules whose inactivation by one or more drugs will selectively kill parasites but not harm the host animal. Because $H$. contortus and related strongylid nematodes are challenging to maintain outside of their hosts, and gene-specific perturbation by double-stranded RNA interference (RNAi) is inconsistent [76], directly assessing gene essentiality on a large scale is not yet practical. However, essentiality can be predicted from functional information (for example, lethality) for C. elegans, and this approach has already yielded credible targets for nematocides [77]. For $H$. contortus, we inferred 641 molecules with essential homologs in C. elegans linked to lethal phenotypes upon gene silencing (see Additional file 1, Table S17). We also screened for enzymatic chokepoints in biological pathways of $H$. contortus. Such chokepoints represent reactions that consume or uniquely produce a molecular compound; the disruption of such enzymes should lead to a toxic accumulation (for unique substrates) or starvation (for unique products) of metabolites within cells $[78,79]$. We gave the highest priority to targets inferred to be encoded by single genes, reasoning that lower allelic variability in $H$. contortus populations would be less likely to give rise to drug resistance. Using this stringent approach, we predicted 260 druggable proteins in $H$. contortus (see Additional file 1, Table S17), of which 106 had ligands fulfilling the Lipinsky rule of five [80] (Table 3). Conspicuous among these were 17 channels or transporters, which represent protein classes known to be targets for anthelmintics, 
Table 3 Druggable candidates encoded in the Haemonchus contortus draft

\begin{tabular}{|c|c|c|}
\hline Group of proteins & Classification (number of molecules) & $\begin{array}{l}\text { Total } \\
\text { number }\end{array}$ \\
\hline Kinases & CAMK (5), TKL (3), tyrosine protein kinases (3), AGC (2), CK1 (2), CMGC (2), STE (1), others (9) & 27 \\
\hline Phosphatases & Fructose-1,6-bisphosphatase I (1), PP2A (1), PP2A-B (1), uridine phosphorylase (1) & 4 \\
\hline GTPases & Rho (1) & 1 \\
\hline Various enzymes & $\begin{array}{l}\text { Replication and repair (9), hydrolases (5), lyases (5), transferases (5), oxidoreductases (3), translation (3), } \\
\text { aminotransferase (2), cellular antigens (2), chaperones and folding catalysts (2), GTP-binding proteins (2), ligases } \\
\text { (2), ubiquitin system (2), cyclins (1), cytoskeleton (1), fatty acid synthase (1), spliceosome (1), others (6) }\end{array}$ & 53 \\
\hline $\begin{array}{l}\text { Transporters and } \\
\text { channels }\end{array}$ & $\begin{array}{l}\text { Primary active transporters (10), incompletely characterized transport systems (3), electrochemical potential-driven } \\
\text { transporters (2), group translocators (2) }\end{array}$ & 17 \\
\hline Transcription factors & Helix-loop-helix (1), helix-turn-helix (4), zinc-coordinating DNA domain (2) & 7 \\
\hline RNAi machinery & Proteins DCR-1 (2) and XPO-1 (1), all involved in small RNA biosynthesis & 3 \\
\hline GPCRs & Class A (1) & 1 \\
\hline
\end{tabular}

including macrocyclic lactones, levamisoles, and AADs $[7,81,82]$, and other candidates including 27 kinases, 7 TFs, and 4 phosphatases known to be specific targets for norcantharidin analogues [77]. This list of prioritized target candidates could be tested for anti-nematodal effects in larval development assays or directly in experimental sheep, and should enable rational anthelmintic design.

\section{Prospects for functional genomics}

Genomic-guided drug discovery would be assisted by assessing essentiality of drug targets directly in $H$. contortus itself. Likewise, functional analysis of the approximately $30 \%$ of $H$. contortus genes that are parasite-specific, some of which are likely to play key roles in host-parasite interactions, would also be enabled by such gene inactivation. However, to date, gene-specific silencing in the parasite itself has been plagued by inconsistent results [76]. Recent findings suggest that this challenge can be overcome if the conditions for effective RNAi were optimized [83,84]. Inconsistent RNAi in $H$. contortus is apparently due to inefficient double-stranded RNA delivery, incomplete knowledge of the RNAi machinery, and variability in gene transcription in different stages or tissues of the parasite. Using the gene set of $H$. contortus, we identified 229 genes encoding proteins involved in the RNAi pathway (Figure 3; see Additional file 1, Table S18), including rde-4 and $r s d-2$, both previously thought to be absent [85], although we did not find $r d e-2$ or sid-2. We also found that most RNAi genes in $H$. contortus are upregulated at the L2, L4, and adult stages (see Additional file 1, Table S18). These findings suggest that future assessments of gene function in $H$. contortus should focus on using these stages, which are most likely to be amenable to RNAi.

\section{Conclusions}

The genomic and transcriptomic exploration of $H$. contortus provides new insights into the molecular biology of one of the most important parasites of small ruminants worldwide. This investigation has elucidated transcriptional alterations taking place throughout the life cycle, particularly during the transition from the free-living to the parasitic stages, and has emphasized molecules involved in host-parasite interactions and immune responses. Determining the genome sequence and transcriptomes of $H$. contortus can accelerate post-genomic explorations of genes and gene products involved in nematode development and reproduction, future proteomic and metabolomic studies, parasite-host interactions, and pathogenesis of disease. The characterization of the RNAi machinery for $H$. contortus also provides a solid platform for functional genomic work in selected stages of the parasite. Therefore, an integrated systems biology approach should provide novel strategies for parasite intervention via drugs, vaccines, and diagnostic tests. For instance, future work could focus on defining a spectrum of key molecules involved in pathways linked to the development of the nervous system in different stages of $H$. contortus, and assessing their potential as drug targets. Moreover, exploring unique groups of molecules, such as SCP/TAPS, TTLs, and the complex array of peptidases, and understanding the roles of these molecules in host-parasite interactions is likely to support the design of new interventions.

The complexity of the genome of $H$. contortus very probably relates to substantial sequence heterogeneity in non-coding regions among individual worms in the populations used for sequencing, and possibly even within individual worms. Although it is unclear why there is so much sequence variation in some genomic regions, it seems that this parasite mutates at a very high rate in non-coding regions, which might explain, then to some extent, the parasite's ability to rapidly become resistant to anthelmintics. To date, much research has focused on investigating possible associations between resistant phenotypes and mutations in particular candidate genes. Having available a draft genome of $H$. contortus now provides a solid foundation for genome-wide studies to identify genetic loci associated with anthelmintic resistance, and to explore their inheritance and mechanisms of drug resistance. Such studies will require an improved understanding of the population 


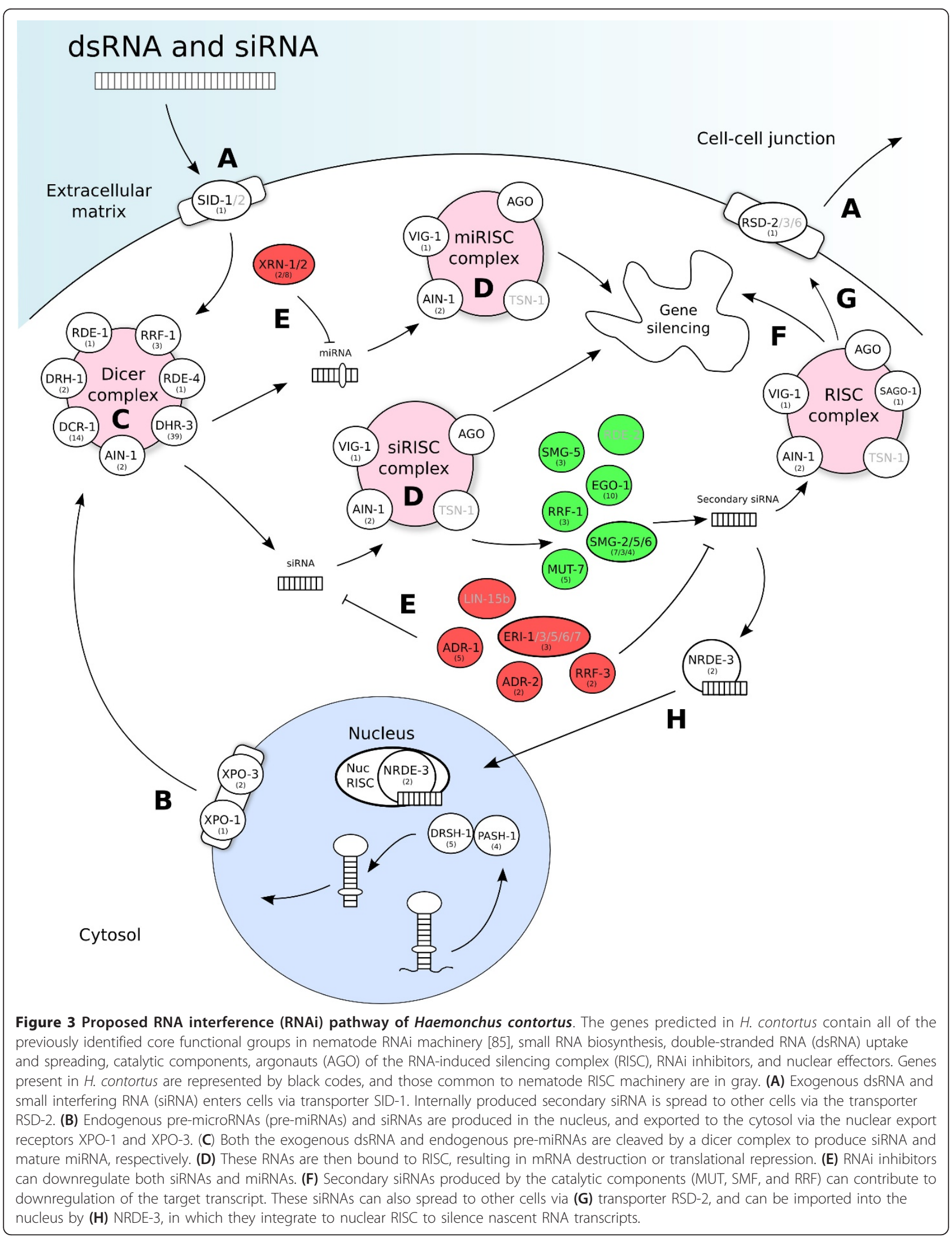


biology and genetics of this parasite, and knowledge of how mutations arise and are inherited. We expect drug resistance in $H$. contortus to be multigenic, and we hypothesize that complex resistance mechanisms operate in this nematode, possibly even involving microRNAs. Clearly, the genome of $H$. contortus will underpin future research in these and many other areas. Although the present study focused on $H$. contortus, the findings and the technological approaches used will be applicable to other parasitic nematodes of major animal and human health importance. Importantly, this first draft genome for a strongylid nematode paves the way for a rapid acceleration in our understanding of a wide range of socioeconomically important parasites of one of the largest nematode orders.

\section{Materials and methods}

\section{Production and procurement of $\mathrm{H}$. contortus}

Animal ethics approval (no. 0707528) was granted by the University of Melbourne. H. contortus was produced in Merino lambs (3 months of age; Victoria, Australia) maintained under helminth-free conditions. Sheep were inoculated intraruminally (via oral intubation) with 5,000 to 10,000 infective L3s of $H$. contortus. Eggs were isolated from the feces of infected sheep ( 1 month after inoculation) using a sucrose flotation procedure [86]. $\mathrm{L} 1 \mathrm{~s}, \mathrm{~L} 2 \mathrm{~s}$, and L3s were produced in culture (at $27^{\circ} \mathrm{C}$ ), as described previously [87]. L1s, L2s, and L3s, identified according to Veglia [4], were collected after 1, 4 and 8 days, respectively, and washed extensively in tap water. L4s and adults of $H$. contortus were collected from the abomasa of infected lambs following euthanasia by intravenous injection of pentobarbitone sodium (Virbac, Carros Cedex, France) 13 days and 1 month, respectively, after infection with L3s. These latter two developmental stages of $H$. contortus were washed extensively in physiological saline, and males and females separated before freezing. All of the developmental stages of $H$. contortus collected were snap-frozen in liquid nitrogen and then stored at $-70^{\circ} \mathrm{C}$ until use.

\section{RNA sequencing and transcriptome assembly}

Total RNA was isolated separately from different developmental stages (egg, L1 to L4, and adult) and sexes (male and female) of $H$. contortus (Haecon-5 strain, Australia) using TriPure isolation reagent (Roche Molecular Biochemicals, Mannheim, Germany). For L1 to L3, packed volumes of 20 to $50 \mu \mathrm{l}$ were used, equating to thousands of larvae. For L4 and adult stages, packed volumes of 50 to $200 \mu \mathrm{l}$ were used, usually equating to 100 to 200 worms per aliquot. RNA yields were estimated spectrophotometrically (NanoDrop 1000; NanoDrop/Thermo Scientific Inc., Pittsburgh PA, USA), and the integrity of RNA was verified using a BioAnalyzer 2100 (Agilent Technologies Inc., Wilmington, DE, USA).
RNA sequencing (RNA-seq) was carried out as described previously [88]. The sequences derived from each library representing each stage and sex were assessed for quality, and adaptors removed. Following removal of any potentially contaminating sequences, RNA-seq data for all stages and both sexes were assembled into de novo predicted transcripts using the programs Velvet and Oases [89] or SOAPdenovo [90]. Non-homologous transcripts were first used to train the de novo gene prediction programs SNAP [91] and AUGUSTUS [92], and transcripts were then used to assist the evidence-based prediction of the non-redundant gene set for $H$. contortus.

\section{Genomic sequencing and initial assembly}

High molecular weight genomic DNA was isolated from adult male and female $H$. contortus (McMaster strain, Australia) using an established protocol [93]. The specificity of genomic DNA was verified by automated sequencing of the second internal transcribed spacer (ITS-2) of nuclear ribosomal DNA following PCR amplification from genomic DNA. Total DNA amounts were determined using a Qubit Fluorometer dsDNA HS Kit (Invitrogen), in accordance with the manufacturer's instructions. Genomic DNA integrity was verified by agarose gel electrophoresis and using a 2000 BioAnalyzer (Agilent). Mate-pair genomic libraries (with $300 \mathrm{bp}$ and 500 bp inserts) were built [88], and checked for both size distribution and quality with a 2100 BioAnalyzer (Agilent). Jumping genomic libraries (with 2, 5, and $10 \mathrm{~kb}$ inserts; see Supplementary Table 1) were constructed as described previously [94]. To produce sufficient amounts of DNA for the jumping libraries, 250 to $500 \mathrm{ng}$ of genomic DNA were subjected to whole genome amplification (WGA) using the REPLI-g Midi Kit (Qiagen Inc., Valencia, CA, USA), in accordance with the manufacturer's protocol. All sequencing was carried out on Illumina machines (either GA II or HiSeq; Illumina Inc., San Diego, CA, USA) with $2 \times 75$ or $2 \times 100$ reads for pairedend libraries, and $2 \times 49$ reads for jumping libraries. For all sequencing, reads were exported to FASTQ format [95]. Several steps were taken to enforce read quality. Custom Perl scripts were used to trim the final nucleotide of each read, nucleotides with a quality score of less than 3, or ' $N$ ' residues. Quality-trimmed reads were kept if they were $65 \mathrm{nt}$ or more long from paired-end data or 48 nt or more long from jumping-library data.

We used a modified version of the read-decontamination pipeline of Kumar and Blaxter [96] to rid the genomic and RNA-seq datasets of any possible contaminating sequences of mammalian, bacterial, mycotic, protistan, and plant origins. In brief, genomic and RNA-seq reads were assembled into preliminary contigs using SOAPdenovo, without scaffolding for genomic DNA and using 
oases with scaffolding for RNA-seq. For genomic DNA contigs and cDNA scaffolds, minimum contig sizes of 60 and $200 \mathrm{nt}$, respectively, were accepted.

We mapped reads to the preliminary contigs with the program Bowtie 2 [97,98]. Unlike Kumar and Blaxter [96], we then performed exhaustive MegablastN [99] searches on all contigs (rather than using a random subset) to determine which sequences had likely contaminant status. MegablastN searching was done against opposing custom nematode and contaminant genomic DNA databases: the nematode set represented genomic assemblies from C. elegans, P. pacificus, A. suum (from WormBase WS230), and Ancylostoma ceylanicum (Schwarz EM, unpublished data). The contaminant set included sheep and cow genomic sequences, 1,991 bacterial genomes (including Prevotella ruminicola) from the European Nucleotide Archive (ENA), and a bovine rumen metagenome [100]. Because $A$. ceylanicum is a strongylid nematode parasite, related to $H$. contortus [101], we expected that any $H$. contortus contigs of genuine nematode origin were highly likely to have a better MegablastN hit to $A$. ceylanicum or C. elegans than to any contaminants. Each preliminary $H$. contortus contig was thus classed as a contaminant if it had a score against the contaminant database of 50 bits or more, and which was at least 50 bits higher than any match by that contig against the nematode database.

We exported all reads that failed to map to a contaminant contig (including both the reads that mapped to non-contaminant contigs and those that did not map to a contig at all). This set of reads was then used for genome and transcriptome assembly, and for quantifying transcription levels. Although our pipeline for decontamination is similar to that of Kumar and Blaxter [96] and uses much of the same source code, it differs by not trying to classify contigs as contaminants based on GC percentage or coverage levels (which we found not to work well with our data), but by using exhaustive MegablastN searching instead.

Our genomic reads, even after initial quality filtering, could not be assembled with Velvet because they required more than $256 \mathrm{~GB}$ of system RAM, the maximum amount available to us on our largest server. Therefore, for Velvet assembly, we used khmer to digitally normalize read frequencies [11]. First, we constructed a hash table of $75 \mathrm{~GB}$ in size, scanned through the paired-end genomic reads, and discarded reads with 20-mers that we had already found 50 times in previous reads. We rescanned the reads, discarding those with unique 20 -mers, reasoning that unique 20-mers in such a large dataset were likely to represent sequencing errors or trace contaminants; khmer estimated the false positive rate of the hash table to be less than 0.001 . The khmer filtering automatically converted the reads from FASTQ to FASTA format. We assembled khmer-filtered reads into a $H$. contortus genome sequence with Velvet 1.2 [102]. For our final Velvet assembly, velveth was run with $k=21$; for preliminary assemblies, velveth was run with values from $\mathrm{k}=41$ down to $\mathrm{k}=19$. The velvetg parameters were as follows: -shortMatePaired3 yes -shortMatePaired4 yes -shortMatePaired5 yes cov_cutoff 4 -exp_cov 100 -min_contig_lgth 200 -ins_length 300 -ins_length_sd 50 -ins_length2 500 -ins_length2_sd 200 -ins_length3 2000 -ins_length4 5000 -ins_length5 10000. Because Velvet 1.2 prioritizes paired-end over jumpinglibrary data, artifactual long-range connections were less likely to confound assembly.

Preliminary assemblies with k-mer values at or near 41 were approximately $700 \mathrm{Mb}$ in size, more than twice the estimate of the genome size $(315 \mathrm{Mb})$ of $H$. contortus based on Feulgen image analysis densitometry [103]. The khmer filtering allowed us to complete assemblies with $\mathrm{k}$ values as low as 21 . With decontaminated reads, $\mathrm{k}=21$ resulted in an assembly size of $404 \mathrm{Mb}$, perhaps because using unusually small word values allowed Velvet's de Bruin graph to merge polymorphisms rather than treat them as distinct, allelic sequences; scaffold N50 was 13.6 $\mathrm{kb}$. The $\mathrm{k}=21$ assembly showed relatively low levels of non-scaffolding residues (79.3\% non-N), but we improved this percentage to $95.8 \%$ non- $\mathrm{N}$ residues by adding reads to the assembly with GapCloser from SOAPdenovo. GapCloser also modestly increased the assembly size to $414 \mathrm{Mb}$ and scaffold N50 to $17.6 \mathrm{~kb}$.

To improve the Velvet assembly, we used SOAPdenovo (version 2.0) to scaffold the $404 \mathrm{Mb}$ assembly using errorcorrected reads without khmer filtering. The program GapCloser was used to close gaps in the scaffolded assembly. With $\mathrm{k}=21$, this gave us an assembly of size $453 \mathrm{Mb}$ that achieved an N50 of $34.2 \mathrm{~kb}$ after gap-closure, with 93.8\% non-N residues. For both Velvet and SOAPdenovo, we tested the gap-filled $\mathrm{k}=21$ assemblies using the program CEGMA [104]. For Velvet, we predicted 157 of 248 conserved eukaryotic genes (CEGs; 63\%) completely, and 211 of $248(85 \%)$ at least partially. Using SOAPdenovo, we predicted 182 of 248 CEGs (73\%) completely, and 232 of $248(91.5 \%)$ at least partially. Given the superior N50, completeness of assembly and the prediction of more CEGs, we selected the SOAPdenovo scaffolded assembly.

\section{Final draft genome assembly}

The initial assembly (453 Mb) was substantially larger than the genome size estimate based on Feulgen image analysis densitometry (315 Mb). Therefore, we re-evaluated the genomic sequence composition by comparing assembled DNA scaffolds containing at least one predicted proteincoding gene against assembled DNA scaffolds that had no such prediction. For this comparison, we did not use only the approximately 26,000 protein-coding genes in our final set, which all had RNA-seq evidence to support their 
expression, but instead used a larger set of approximately 29,184 genes, which included both RNA-seq-supported and protein-supported predictions. Moreover, we mapped Illumina sequencing reads that had been decontaminated but not yet subjected to digital normalization with khmer, so that variations in sequencing coverage would remain detectable. We found that scaffolds containing proteincoding genes had a much higher coverage of Illumina sequencing reads (with a single distinct peak) than scaffolds that were completely devoid of predicted proteincoding genes (which were represented by a very lowcoverage peak). All the high-coverage coding regions were contained within a total of $320 \mathrm{Mb}$ of scaffolded sequences, whereas all of the low-coverage non-coding regions were within a total of $133 \mathrm{Mb}$ of scaffolded sequences. Moreover, when we examined the two sequence sets with CEGMA for completeness of gene content, the $320 \mathrm{Mb}$ set was essentially identical to the $453 \mathrm{Mb}$ assembly, whereas the $133 \mathrm{Mb}$ set was almost completely devoid of gene content. We therefore selected the $320 \mathrm{Mb}$ scaffold set as our final draft assembly. Lowcoverage scaffolds might represent a residue from the khmer removal of sequences with high coverage, and heterozygosity/heterogeneity/haplotype differences linked to non-coding regions, possibly due to variations among individual worms of the population.

\section{Identification and annotation of non-coding regions and protein-coding genes}

Genomic repeats specific to $H$. contortus were modeled using the program RepeatModeler [105] by merging repeat predictions by RECON [106] and RepeatScout [107]. Repeats in the $H$. contortus genome assembly were identified by RepeatMasker [108] using modeled repeats (via RepeatModeler) and known repeats in Repbase (version 17.02) [109].

The $H$. contortus protein-coding gene set was inferred using an integrative approach, utilizing the transcriptomic data for all stages and both sexes sequenced in the present study. First, all 185,706 contigs representing the combined transcriptome for $H$. contortus were run through BLAT [110] and filtered for full-length open reading frames (ORFs), ensuring the validity of splice sites. These ORFs were then used to train the de novo gene prediction programs SNAP [91] and AUGUSTUS [92] by producing a hidden Markov model (HMM) for each program. The same ORFs were also given (as an expressed sequence tag (EST) input) to MAKER2 [111] to provide evidence for predicted genes. In addition, all raw reads representing the combined $H$. contortus transcriptome were run through the programs TopHat and Cufflinks to provide additional information on transcripts and on exon-intron boundaries in the form of a Generic Feature Format (GFF) file.
HMMs, the EST input, and the GFF file were subjected to analysis using MAKER2 to provide a consensus set of 27,782 genes for $H$. contortus. Genes inferred to encode peptides of 30 or more amino acids in length were preserved, resulting in the prediction of a total of 27,135 genes. To account for the genes in DNA repeat regions, identified by RepeatMasker, we removed genes $(n=1,028)$ that overlapped these regions by at least one nucleotide and did not have a similarity match (BLASTp; e-value $\leq 10^{-5}$ ) [112] with genes of C. elegans. Following filtering of the predicted genes by Annotation Edit Distance (AED $<0.4$ ) [113], the final set was inferred to contain 23,610 protein-coding genes. The predicted genes were represented by amino acid and cDNA sequences.

\section{Functional annotation of all predicted protein sequences}

First, following prediction of the protein-coding gene set for $H$. contortus, each inferred amino acid sequence was assessed for conserved protein domains using InterProScan $[114,115]$, employing default settings. Second, amino acid sequences were subjected to BLASTp (e-value $\leq 10^{-5}$ ) against the following protein databases: C. elegans in WormBase [116]; Swiss-Prot and TrEMBL within UniProtKB [117]; Kinase SARfari [118] and the protein kinase database for C. elegans [119], which contains all domain information for C. elegans kinases [120]; GPCR SARfari [118]; Transporter Classification Database [121,122]; KEGG [123,124]; LGICs [125]; ChEMBL [126]; NCBI protein $\mathrm{nr}$ [127]; and an in-house RNAi machinery database for nematodes. Finally, the BLASTp results were used to infer key protein groups, including peptidases, kinases, phosphatases, GTPases, GPCRs, channel and transporter proteins, TFs, major sperm proteins, vitellogenins, SCP/ TAPS proteins, and RNAi machinery proteins.

Each coding gene was assessed against the known KEGG Orthology (KO) term BLAST hits. These BLAST hits were clustered to a known protein family using the KEGGBRITE hierarchy in a custom script. ES proteins were predicted using SignalP (version 4.0) [128] and TMHMM (version 2.0c) $[122,129,130]$ and by BLASTp homology searching of the validated Signal Peptide Database [131] and of an ES database containing published proteomic data for A. suum [14], B. malayi [15]. C. elegans [116], and T. spiralis [16]. In the final annotation, proteins inferred from genes were classified based on a homology match (e-value cut-off, $\leq 10^{-5}$ ) to: (i) a curated, specialist protein database, followed by (ii) the KEGG database, followed by (iii) the Swiss-Prot database, followed by (iv) the annotated gene set for a model organism, including C. elegans, followed by (v) a recognized, conserved protein domain based on InterProScan analysis. Any inferred proteins lacking a match (e-value cut-off, $\leq 10^{-5}$ ) in at least one of these analyses were designated hypothetical proteins. The 
final annotated protein-coding gene set for $H$. contortus is available for download at WormBase [116] in nucleotide and amino acid formats.

\section{Differential transcription analysis}

The analysis of empirical RNA-seq data for the developmental stages and sexes of $H$. contortus was conducted using edge $R$ [132], an $R$ programming language [133] package. Trimmomatic software [134], using the parameters phred64, ILLUMINACLIP:illuminaClipping. fa:2:40:20,LEADING:3, TRAILING:3, SLIDINGWINDOW:4:20, MINLEN:40, was used to filter the pairedend RNA-seq reads for quality in individual samples (representing egg, L1, L2, L3, and L4 males, L4 females, and adult males and adult females).

Each set of the decontaminated and quality-filtered paired-end RNA-seq data was mapped to the set of cDNAs using Burrows-Wheeler Aligner software [135]. The numbers of mapped reads per individual gene were extracted using the program SAMtools [136]. The resultant read counts per developmental stage were used as input data for edgeR. Initially, the levels of differential transcription data were calculated by pairwise comparison of stages in the life cycle of $H$. contortus (for example, egg versus L1; L1 versus L2; L2 versus L3; L3 versus L4; L3 versus adult; L4 versus adult) and of sexes (L4 female versus L4 male; adult female versus adult male). Using edgeR dispersion factor zero, the genes were considered differentially transcribed if the logarithmic change in fold change (FC) compared with the normalized read count data was greater than or equal to 2 and the $P$-value was less than or equal to $10^{-5}$. The levels of differential transcription data were then calculated by pairwise comparisons between all free-living (egg, L1, L2. and L3) and parasitic (L4 and adult) stages. The genes were considered differentially transcribed, using edgeR-calculated common and genewise dispersion factors, if the FC compared with the normalized read count data was greater than or equal to 2 and the false discovery rate (FDR) was less than or equal to 0.05 . To identify abundant sex-enriched genes in adult $H$. contortus, more stringent criteria ( $\mathrm{FC} \geq 4 ; \mathrm{FDR} \leq 0.05)$ were applied. The resultant differentially transcribed genes were subjected to genetic interaction network analysis $[33,34]$, based on the pre-calculated, weighed interactions among C. elegans genes. Hubs with at least 10 interactions (degree $\geq 10$ ) among different genes were considered significant.

\section{Protein homology}

Homologs between $H$. contortus and A. suum, B. malayi, C. elegans, and T. spiralis were inferred by comparison of all proteins by BLASTp (e-value $\leq 10^{-5}$ ), pairing proteins based on reciprocal best hits, and inferring homologous groups from pairs using a custom script.
Prediction of essentiality, chokepoints, and drug targets Essentiality was inferred by filtering C. elegans homologs (BLASTp; e-value $\leq 10^{-5}$ ) [112] representing lethal phenotype in RNAi experiments listed in WormBase release WS222 [116]. The metabolic chokepoints were predicted from essential genes with a unique match to the combined identifier of KEGG pathway and KO group. KEGG pathways and $\mathrm{KO}$ groups were inferred from the KEGG database (BLASTp; e-value $\leq 10^{-5}$ ). The molecules in metabolic chokepoints that satisfied Lipinski's rule of five in ChEMBL were identified from matches with target molecules (BLASTp; e-value $\leq 10^{-30}$ ) in the ChEMBL database.

\section{Additional bioinformatic and data analyses, and use of software for document preparation}

Data analysis was conducted in a Unix environment or Microsoft Excel 2007 using standard commands. Bioinformatic scripts required to facilitate data analysis were designed using mainly the Python 2.6 scripting language.

\section{Data availability}

The genomic sequence and gene predictions for $H$. contortus are available in WormBase. The genome sequence has also been deposited at DDBJ/EMBL/GenBank (accession number AUUS00000000), and genomic and RNA-seq reads in the NCBI short read archive (SRA; accession numbers SRP027504 and SRP026668, respectively).

\section{Additional material}

Additional file 1: Supplementary tables S1-S18.

\section{Abbreviations}

AAD: aminoacetonitrile derivative; AED: Annotation Edit Distance; ASP: Ancylostoma-secreted protein; CEGMA: Core Eukaryotic Genes Mapping Approach; ES: Excretory/secretory; EST: expressed sequence tag; FC: fold change; FDR: false discovery rate; GFF: Generic Feature Format; GPCR: G protein-coupled receptor; H-gal-GP: Haemonchus galactose-containing glycoprotein complex; HMM: hidden Markov model; KEGG: Kyoto Encyclopedia of Genes and Genomes; KO: KEGG Orthology; L1 to L4: Firststage to fourth-stage larvae; LGIC: Ligand-gated ion channel; LINE: long interspersed nuclear element; LTR: long terminal repeat; NIF: Neutrophil inhibitory factor; ORF: open reading frame; RTE: retrotransposable element; RNAi: RNA interference; SCP/TAPS: Sperm-coating protein/Tpx-1/Ag5/PR-1/ Sc7; SINE: short interspersed nuclear element; TF: Transcription factor; Th: T helper; TTL: transthyretin-like; VIC: voltage-gated channel.

\section{Authors' contributions}

RBG, BEC, and AJ produced $H$. contortus in sheep and isolated genomic DNA; BEC and NDY isolated RNA; BAW constructed RNA-seq libraries; IA optimized Illumina protocols for paired-end libraries. CTB, ACH, and JP devised khmer filtering; CTB and EMS ran it on quality-filtered genomic reads. EMS decontaminated all reads in silico. EMS and PKK performed genomic assembly and annotation; PKK, BEC, NDY, ARJ, and RSH performed transcriptomic analyses. TRG estimated the genome size of $H$. contortus by Feulgen image analysis densitometry. RBG, EMS, PKK, and PWS wrote the 
manuscript, with crucial contributions from NDY, ARJ, and inputs from XQZ PRB, and other co-authors. RBG and PWS managed the project. All authors read and approved the final manuscript.

\section{Acknowledgements}

This paper is dedicated to the memories of Sue Newton and Paul J Presidente. We thank Sujai Kumar and Mark Blaxter for providing methods for removing contaminant sequences, and Ali Mortazavi for early sequence analyses. We are grateful to Jody Zawadzki and the late Paul Presidente for originally providing us with the McMaster and Haecon-5 strains of $H$. contortus. This project was funded by the Australian Research Council (RBG and PWS), National Health and Medical Research Council (NHMRC), Howard Hughes Medical Institute, and the National Institutes of Health. This project was also supported by a Victorian Life Sciences Computation Initiative (grant number VR0007) on its Peak Computing Facility at the University of Melbourne, an initiative of the Victorian Government. Other support from the Australian Academy of Science, the Australian-American Fulbright Commission, Alexander von Humboldt Foundation, Melbourne Water Corporation, and the IBM Research Collaboratory for Life Sciences Melbourne is gratefully acknowledged. NDY is an NHMRC Early Career Research Fellow. We also acknowledge the contributions of all staff at WormBase. We thank the anonymous reviewers for their very constructive reports on our original manuscript.

\section{Authors' details}

${ }^{1}$ Howard Hughes Medical Institute and Division of Biology, California Institute of Technology, Pasadena, California 91125, USA. ${ }^{2}$ Faculty of Veterinary Science, The University of Melbourne, Corner of Flemington Road and Park Drive, Parkville, Victoria 3010, Australia. ${ }^{3}$ Current address: Department of Molecular Biology and Genetics, Cornell University, Ithaca, New York, 14853-2703, USA. ${ }^{4}$ Department of Microbiology and Molecular Genetics, Michigan State University, East Lansing, Michigan, 48824, USA. ${ }^{5}$ Department of Computer Science and Engineering, Michigan State University, East Lansing, Michigan, 48824, USA. ${ }^{6}$ Eskitis Institute for Cell and Molecular Therapies, Griffith University, N75 Don Young Road, Brisbane Innovation Park, Nathan, Queensland 4111, Australia. ${ }^{7}$ Faculty of Medicine, Nursing and Health Sciences, Monash University, Wellington Road, Clayton, Victoria 3800, Australia. ${ }^{8}$ State Key Laboratory of Veterinary Etiological Biology, Lanzhou Veterinary Research Institute, Chinese Academy of Agricultural Sciences, 1 Xujiaping, Yanchangbu, Lanzhou, Gansu Province 730046, PR China. ${ }^{9}$ Department of Integrative Biology, University of Guelph, Ontario, Canada N1G 2W1. ${ }^{10}$ Center for Biodiscovery and Molecular Development of Therapeutics, Queensland Tropical Health Alliance, James Cook University, Cairns, Queensland 4870, Australia.

Received: 10 April 2013 Revised: 8 August 2013

Accepted: 28 August 2013 Published: 28 August 2013

\section{References}

1. Bethony JM, Loukas A, Hotez PJ, Knox DP: Vaccines against blood-feeding nematodes of humans and livestock. Parasitology 2006, 133(Suppl):S63-79

2. Nikolaou S, Gasser RB: Prospects for exploring molecular developmental processes in Haemonchus contortus. Int J Parasitol 2006, 36:859-868.

3. Scott I, Sutherland I: Gastrointestinal Nematodes of Sheep and Cattle: Biology and Control Oxford: Wiley-Blackwell; 2010.

4. Veglia F: The anatomy and life-history of the Haemonchus contortus (Rud.). Rep Dir Vet Res 1915, 3-4:347-500.

5. Kaplan RM, Vidyashankar AN: An inconvenient global truth worming and anthelmintic resistance. Vet Parasitol 2012, 186:70-78.

6. Knox D: Proteases in blood-feeding nematodes and their potential as vaccine candidates. Adv Exp Med Biol 2011, 712:155-176.

7. Kaminsky R, Ducray P, Jung M, Clover R, Rufener L, Bouvier J, Weber SS, Wenger A, Wieland-Berghausen S, Goebel T, Gauvry N, Pautrat F, Skripsky T, Froelich O, Komoin-Oka C, Westlund B, Sluder A, Mäser P: A new class of anthelmintics effective against drug-resistant nematodes. Nature 2008, 452:176-180.

8. von Samson-Himmelstjerna G, Harder A, Sangster NC, Coles GC: Efficacy of two cyclooctadepsipeptides, PF1022A and emodepside, against anthelmintic-resistant nematodes in sheep and cattle. Parasitology 2005, 130:343-347.
9. Little PR, Hodge A, Maeder SJ, Wirtherle NC, Nicholas DR, Cox GG, Conder GA: Efficacy of a combined oral formulation of derquantelabamectin against the adult and larval stages of nematodes in sheep, including anthelmintic-resistant strains. Vet Parasitol 2011, 181:180-193.

10. Pell J, Hintze A, Canino-Koning R, Howe A, Tiedje JM, Brown CT: Scaling metagenome sequence assembly with probabilistic de Bruijn graphs. Proc Natl Acad Sci USA 2012, 109:13272-13277.

11. Brown $C T$, Howe A, Zhang $Q$, Pyrkosz AB, Brom TH: A reference-free algorithm for computational normalization of shotgun sequencing data. NASA ADS 2012, eprint arXiv:1203.4802 [q-bio.GN].

12. C elegans, Sequencing Consortium C: Genome sequence of the nematode C. elegans a platform for investigating biology. Science 1998, 282:2012-2018.

13. Dieterich C, Clifton SW, Schuster LN, Chinwalla A, Delehaunty K, Dinkelacker I, Fulton L, Fulton R, Godfrey J, Minx P, Mitreva M, Roeseler W, Tian H, Witte H, Yang SP, Wilson RK, Sommer RJ: The Pristionchus pacificus genome provides a unique perspective on nematode lifestyle and parasitism. Nat Genet 2008, 40:1193-1198.

14. Jex AR, Liu S, Li B, Young ND, Hall RS, Li Y, Yang L, Zeng N, Xu X, Xiong Z, Chen F, Wu X, Zhang G, Fang X, Kang Y, Anderson GA, Harris TW, Campbell BE, Vlaminck J, Wang T, Cantacessi C, Schwarz EM, Ranganathan S, Geldhof P, Nejsum P, Sternberg PW, Yang H, Wang J, Wang J, Gasser RB: Ascaris suum draft genome. Nature 2011, 479:529-533.

15. Ghedin E, Wang S, Spiro D, Caler E, Zhao Q, Crabtree J, Allen JE, Delcher AL, Guiliano DB, Miranda-Saavedra D, Angiuoli SV, Creasy T, Amedeo P, Haas B, El-Sayed NM, Wortman JR, Feldblyum T, Tallon L, Schatz M, Shumway M, Koo H, Salzberg SL, Schobel S, Pertea M, Pop M, White O, Barton GJ, Carlow CK, Crawford MJ, Daub J, et al: Draft genome of the filarial nematode parasite Brugia malayi. Science 2007, 317:1756-1760.

16. Mitreva M, Jasmer DP, Zarlenga DS, Wang Z, Abubucker S, Martin J, Taylor CM, Yin Y, Fulton L, Minx P, Yang SP, Warren WC, Fulton RS, Bhonagiri V, Zhang X, Hallsworth-Pepin K, Clifton SW, McCarter JP, Appleton J, Mardis ER, Wilson RK: The draft genome of the parasitic nematode Trichinella spiralis. Nat Genet 2011, 43:228-235.

17. Spanier B, Sturzenbaum SR, Holden-Dye LM, Baumeister R: Caenorhabditis elegans neprilysin NEP-1: an effector of locomotion and pharyngeal pumping. J Mol Biol 2005, 352:429-437.

18. McKerrow JH, Caffrey C, Kelly B, Loke P, Sajid M: Proteases in parasitic diseases. Annu Rev Pathol 2006, 1:497-536

19. Hewitson JP, Grainger JR, Maizels RM: Helminth immunoregulation: the role of parasite secreted proteins in modulating host immunity. Mol Biochem Parasitol 2009, 167:1-11.

20. Baugh LR, Demodena J, Sternberg PW: RNA Pol II accumulates at promoters of growth genes during developmental arrest. Science 2009, 324:92-94.

21. Cox GN, Shamansky LM, Boisvenue RJ: Haemonchus contortus: evidence that the $3 \mathrm{~A} 3$ collagen gene is a member of an evolutionarily conserved family of nematode cuticle collagens. Exp Parasitol 1990, 70:175-185.

22. Pratt $D, C o x G N$, Milhausen MJ, Boisvenue RJ: A developmentally regulated cysteine protease gene family in Haemonchus contortus. Mol Biochem Parasitol 1990, 43:181-191.

23. Williamson AL, Lecchi P, Turk BE, Choe Y, Hotez PJ, McKerrow JH, Cantley LC, Sajid M, Craik CS, Loukas A: A multi-enzyme cascade of hemoglobin proteolysis in the intestine of blood-feeding hookworms. J Biol Chem 2004, 279:35950-35957.

24. Ranjit N, Zhan B, Stenzel DJ, Mulvenna J, Fujiwara R, Hotez PJ, Loukas A: A family of cathepsin $B$ cysteine proteases expressed in the gut of the human hookworm, Necator americanus. Mol Biochem Parasitol 2008, 160:90-99.

25. Ranjit N, Zhan B, Hamilton B, Stenzel D, Lowther J, Pearson M, Gorman J, Hotez P, Loukas A: Proteolytic degradation of hemoglobin in the intestine of the human hookworm Necator americanus. J Infect Dis 2009, 199:904-912

26. Roos MH, Tielens AG: Differential expression of two succinate dehydrogenase subunit- $B$ genes and a transition in energy metabolism during the development of the parasitic nematode Haemonchus contortus. Mol Biochem Parasitol 1994, 66:273-281.

27. Skuce PJ, Stewart EM, Smith WD, Knox DP: Cloning and characterization of glutamate dehydrogenase (GDH) from the gut of Haemonchus contortus. Parasitology 1999, 118:297-304. 
28. Fetterer $\mathrm{RH}$, Hill DE, Rhoads ML: Characterization of a hemoglobin-like protein from adult Haemonchus contortus. J Parasitol 1999, 85:295-300.

29. Blaxter ML: Nemoglobins divergent nematode globins. Parasitol Today 1993, 9:353-360.

30. Liddell S, Knox DP: Extracellular and cytoplasmic $\mathrm{Cu} / \mathrm{Zn}$ superoxide dismutases from Haemonchus contortus. Parasitology 1998, 116(Pt 4):383-394.

31. Kotze AC: Catalase induction protects Haemonchus contortus against hydrogen peroxide in vitro. Int J Parasitol 2003, 33:393-400.

32. van Rossum AJ, Jefferies JR, Rijsewijk FA, LaCourse EJ, Teesdale-Spittle $P$, Barrett J, Tait A, Brophy PM: Binding of hematin by a new class of glutathione transferase from the blood-feeding parasitic nematode Haemonchus contortus. Infect Immun 2004, 72:2780-2790

33. Zhong W, Sternberg PW: Genome-wide prediction of $C$. elegans genetic interactions. Science 2006, 311:1481-1484.

34. Lee I, Lehner B, Crombie C, Wong W, Fraser AG, Marcotte EM: A single gene network accurately predicts phenotypic effects of gene perturbation in Caenorhabditis elegans. Nat Genet 2008, 40:181-188.

35. Cantacessi C, Campbell BE, Visser A, Geldhof P, Nolan MJ, Nisbet AJ, Matthews JB, Loukas A, Hofmann A, Otranto D, Sternberg PW, Gasser RB: A portrait of the "SCP/TAPS" proteins of eukaryotes-developing a framework for fundamental research and biotechnological outcomes. Biotechnol Adv 2009, 27:376-388.

36. Hawdon JM, Jones BF, Hoffman DR, Hotez PJ: Cloning and characterization of Ancylostoma-secreted protein. A novel protein associated with the transition to parasitism by infective hookworm larvae. J Biol Chem 1996, 271:6672-6678.

37. Datu BJ, Loukas A, Cantacessi C, O'Donoghue P, Gasser RB: Investigation of the regulation of transcriptional changes in Ancylostoma caninum larvae following serum activation, with a focus on the insulin-like signalling pathway. Vet Parasitol 2009, 159:139-148.

38. Schallig HD, van Leeuwen MA, Cornelissen AW: Protective immunity induced by vaccination with two Haemonchus contortus excretory secretory proteins in sheep. Parasite Immunol 1997, 19:447-453.

39. Rehman A, Jasmer DP: A tissue specific approach for analysis of membrane and secreted protein antigens from Haemonchus contortus gut and its application to diverse nematode species. Mol Biochem Parasitol 1998, 97:55-68.

40. Yatsuda AP, Krijgsveld J, Cornelissen AW, Heck AJ, de Vries E: Comprehensive analysis of the secreted proteins of the parasite Haemonchus contortus reveals extensive sequence variation and differential immune recognition. J Biol Chem 2003, 278:16941-16951.

41. Bethony JM, Simon G, Diemert DJ, Parenti D, Desrosiers A, Schuck S, Fujiwara R, Santiago H, Hotez PJ: Randomized, placebo-controlled, double-blind trial of the Na-ASP-2 hookworm vaccine in unexposed adults. Vaccine 2008, 26:2408-2417.

42. Diemert DJ, Pinto AG, Freire J, Jariwala A, Santiago $H$, Hamilton RG, Periago MV, Loukas A, Tribolet L, Mulvenna J, Correa-Oliveira R, Hotez PJ, Bethony JM: Generalized urticaria induced by the Na-ASP-2 hookworm vaccine: implications for the development of vaccines against helminths. J Allergy Clin Immunol 2012, 130:169-176, e166.

43. Asojo OA, Goud G, Dhar K, Loukas A, Zhan B, Deumic V, Liu S, Borgstahl GE, Hotez PJ: X-ray structure of Na-ASP-2, a pathogenesis-related-1 protein from the nematode parasite, Necator americanus, and a vaccine antigen for human hookworm infection. J Mol Biol 2005, 346:801-814.

44. Moyle M, Foster DL, McGrath DE, et al: A hookworm glycoprotein that inhibits neutrophil function is a ligand of the integrin CD11b/CD18. J Biol Chem 1994, 269:10008-10015.

45. Rieu P, Sugimori T, Griffith DL, Arnaout MA: Solvent-accessible residues on the metal ion-dependent adhesion site face of integrin CR3 mediate its binding to the neutrophil inhibitory factor. J Biol Chem 1996, 271:15858-15861.

46. Geldhof P, Whitton C, Gregory WF, Blaxter M, Knox DP: Characterisation of the two most abundant genes in the Haemonchus contortus expressed sequence tag dataset. Int J Parasitol 2005, 35:513-522.

47. Skuce PJ, Yaga R, Lainson FA, Knox DP: An evaluation of serial analysis of gene expression (SAGE) in the parasitic nematode, Haemonchus contortus. Parasitology 2005, 130:553-559.

48. Rahat O, Yitzhaky A, Schreiber G: Cluster conservation as a novel tool for studying protein-protein interactions evolution. Proteins 2008, 71:621-630.
49. Lee $Y$, Lee DH, Kho CW, Lee AY, Jang M, Cho S, Lee CH, Lee JS, Myung PK, Park BC, Park SG: Transthyretin-related proteins function to facilitate the hydrolysis of 5-hydroxyisourate, the end product of the uricase reaction. FEBS Lett 2005, 579:4769-4774.

50. Ramazzina I, Folli C, Secchi A, Berni R, Percudani R: Completing the uric acid degradation pathway through phylogenetic comparison of whole genomes. Nat Chem Biol 2006, 2:144-148.

51. Li X, Buxbaum JN: Transthyretin and the brain re-visited is neuronal synthesis of transthyretin protective in Alzheimer's disease? Mol Neurodegener 2011, 6:79.

52. Wang X, Li W, Zhao D, Liu B, Shi Y, Chen B, Yang H, Guo P, Geng X, Shang Z, Peden E, Kage-Nakadai E, Mitani S, Xue D: Caenorhabditis elegans transthyretin-like protein TTR-52 mediates recognition of apoptotic cells by the CED-1 phagocyte receptor. Nat Cell Biol 2010, 12:655-664.

53. Jacob J, Vanholme B, Haegeman A, Gheysen G: Four transthyretin-like genes of the migratory plant-parasitic nematode Radopholus similis members of an extensive nematode-specific family. Gene 2007, 402:9-19.

54. Parkinson J, Mitreva M, Whitton C, Thomson M, Daub J, Martin J, Schmid R, Hall N, Barrell B, Waterston RH, McCarter JP, Blaxter ML: A transcriptomic analysis of the phylum Nematoda. Nat Genet 2004, 36:1259-1267.

55. Vercauteren I, Geldhof P, Peelaers I, Claerebout E, Berx G, Vercruysse J: Identification of excretory-secretory products of larval and adult Ostertagia ostertagi by immunoscreening of cDNA libraries. Mol Biochem Parasitol 2003, 126:201-208.

56. Saverwyns H, Visser A, Van Durme J, Power D, Morgado I, Kennedy MW, Knox DP, Schymkowitz J, Rousseau F, Gevaert K, Vercruysse J, Claerbout E, Geldhof $P$ : Analysis of the transthyretin-like (TL) gene family in Ostertagia ostertagi-comparison with other strongylid nematodes and Caenorhabditis elegans. Int J Parasitol 2008, 38:1545-1556.

57. Hewitson JP, Harcus YM, Curwen RS, Dowle AA, Atmadja AK, Ashton PD, Wilson A, Maizels RM: The secretome of the filarial parasite, Brugia malayi: proteomic profile of adult excretory-secretory products. $\mathrm{Mol}$ Biochem Parasitol 2008, 160:8-21.

58. Gao B, Allen R, Maier T, Davis EL, Baum TJ, Hussey RS: The parasitome of the phytonematode Heterodera glycines. MPMI 2003, 16:720-726.

59. McCarter JP, Mitreva MD, Martin J, Dante M, Wylie T, al. e: Analysis and functional classification of transcripts from the nematode Meloidogyne incognita. Genome Biol 2004, 4:R26.

60. Furlanetto C, Cardle L, Brown DJF, Jones JT: Analysis of expressed sequence tags from the ectoparasitic nematode Xiphinema index. Nematology 2005, 7:95-104.

61. Mulvenna J, Hamilton B, Nagaraj SH, Smyth D, Loukas A, Gorman JJ: Proteomics analysis of the excretory/secretory component of the bloodfeeding stage of the hookworm, Ancylostoma caninum. Mol Cell Proteomics 2009, 8:109-121.

62. Munn EA: A helical, polymeric extracellular protein associated with the luminal surface of Haemonchus contortus intestinal cells. Tissue Cell 1977, 9:23-34.

63. Newton SE, Munn EA: The development of vaccines against gastrointestinal nematode parasites, particularly Haemonchus contortus. Parasitol Today 1999, 15:116-122.

64. Smith SK, Smith WD: Immunisation of sheep with an integral membrane glycoprotein complex of Haemonchus contortus and with its major polypeptide components. Res Vet Sci 1996, 60:1-6.

65. Redmond DL, Knox DP, Newlands G, Smith WD: Molecular cloning and characterisation of a developmentally regulated putative metallopeptidase present in a host protective extract of Haemonchus contortus. Mol Biochem Parasitol 1997, 85:77-87.

66. Newlands GF, Skuce PJ, Nisbet AJ, Redmond DL, Smith SK, Pettit D, Smith WD: Molecular characterization of a family of metalloendopeptidases from the intestinal brush border of Haemonchus contortus. Parasitology 2006, 133:357-368.

67. Longbottom D, Redmond DL, Russell M, Liddell S, Smith WD, Knox DP: Molecular cloning and characterisation of a putative aspartyl proteinase associated with a gut membrane protein complex from adult Haemonchus contortus. Mol Biochem Parasitol 1997, 88:63-72.

68. Smith WD, Skuce PJ, Newlands GFJ, Smith SK, Pettit D: Aspartyl proteases from the intestinal brush border of Haemonchus contortus as protective antigens for sheep. Parasite Immunol 2003, 25:521-530. 
69. Cox GN, Pratt D, Hageman R, Boisvenue RJ: Molecular cloning and primary sequence of a cysteine protease expressed by Haemonchus contortus adult worms. Mol Biochem Parasitol 1990, 41:25-34.

70. Pratt D, Armes LG, Hageman R, Reynolds V, Boisvenue RJ, Cox GN: Cloning and sequence comparisons of four distinct cysteine proteases expressed by Haemonchus contortus adult worms. Mol Biochem Parasitol 1992, 51:209-218.

71. Skuce PJ, Redmond DL, Liddell S, Stewart EM, Newlands GF, Smith WD, Knox DP: Molecular cloning and characterization of gut-derived cysteine proteinases associated with a host protective extract from Haemonchus contortus. Parasitology 1999, 119:405-412.

72. Dalton JP, Brindley PJ, Donnelly S, Robinson MW: The enigmatic asparaginyl endopeptidase of helminth parasites. Trends Parasitol 2009, 25:59-61.

73. Geldhof $P$, Knox D: The intestinal contortin structure in Haemonchus contortus: an immobilised anticoagulant? Int J Parasitol 2008, 38:1579-1588.

74. Harder A, Schmitt-Wrede HP, Krücken J, Marinovski P, Wunderlich F, Willson J, Amliwala K, Holden-Dye L, Walker R: Cyclooctadepsipeptides an anthelmintically active class of compounds exhibiting a novel mode of action. Int J Antimicrob Ag 2003, 22:318-331.

75. Shanmugam D, Ralph SA, Carmona SJ, Crowther GJ, Roos DS, Agüero F: Integrating and Mining Helminth Genomes to Discover and Prioritize Novel Therapeutic Targets Wiley Online Library; 2012, Wiley, USA.

76. Geldhof P, Visser A, Clark D, Saunders G, Britton C, Gilleard J, Berriman M, Knox D: RNA interference in parasitic helminths: current situation, potential pitfalls and future prospects. Parasitology 2007, 134:609-619.

77. Campbell BE, Tarleton M, Gordon CP, Sakoff JA, Gilbert J, McCluskey A, Gasser RB: Norcantharidin analogues with nematocidal activity in Haemonchus contortus. Bioorg Med Chem Lett 2011, 21:3277-3281.

78. Yeh I, Hanekamp T, Tsoka S, Karp PD, Altman RB: Computational analysis of Plasmodium falciparum metabolism: organizing genomic information to facilitate drug discovery. Genome Res 2004, 14:917-924.

79. Berriman M, Haas BJ, LoVerde PT, Wilson RA, Dillon GP, Cerqueira GC, Mashiyama ST, Al-Lazikani B, Andrade LF, Ashton PD, Aslett MA, Bartholomeu DC, Blandin G, Caffrey CR, Coghlan A, Coulson R, Day TA, Delcher A, DeMarco R, Djikeng A, Eyre T, Gamble JA, Ghedin E, Gu Y, HertzFowler C, Hirai H, Hirai Y, Houston R, Ivens A, Johnston DA, et al: The genome of the blood fluke Schistosoma mansoni. Nature 2009, 460:352-U365.

80. Lipinski CA: Lead- and drug-like compounds: the rule-of-five revolution. Drug Discov Today: Technol 2004, 1:337-341.

81. Campbell WC, Fisher MH, Stapley EO, Albers-Schonberg G, Jacob TA: Ivermectin: a potent new antiparasitic agent. Science 1983, 221:823-828.

82. Qian H, Robertson AP, Powell-Coffman JA, Martin RJ: Levamisole resistance resolved at the single-channel level in Caenorhabditis elegans. FASEB J 2008, 22:3247-3254.

83. Samarasinghe $B$, Knox DP, Britton C: Factors affecting susceptibility to RNA interference in Haemonchus contortus and in vivo silencing of an H11 aminopeptidase gene. Int J Parasitol 2011, 41:51-59.

84. Selkirk ME, Huang SC, Knox DP, Britton C: The development of RNA interference (RNAi) in gastrointestinal nematodes. Parasitology 2012 139:605-612

85. Dalzell JJ, McVeigh $P$, Warnock ND, Mitreva M, Bird DM, Abad P Fleming CC, Day TA, Mousley A, Marks NJ, Maule AG: RNAi effector diversity in nematodes. PLOS Negl Trop Dis 2011, 5:e1176.

86. Mes TH, Eysker M, Ploeger HW: A simple, robust and semi-automated parasite egg isolation protocol. Nat Protoc 2007, 2:486-489.

87. Nikolaou S, Hartman D, Presidente PJ, Newton SE, Gasser RB: HcSTK, a Caenorhabditis elegans PAR-1 homologue from the parasitic nematode, Haemonchus contortus. Int J Parasitol 2002, 32:749-758.

88. Mortazavi A, Schwarz EM, Williams B, Schaeffer L, Antoshechkin I, Wold BJ, Sternberg PW: Scaffolding a Caenorhabditis nematode genome with RNAseq. Genome Res 2010, 20:1740-1747.

89. Schulz MH, Zerbino DR, Vingron M, Birney E: Oases: robust de novo RNAseq assembly across the dynamic range of expression levels. Bioinformatics 2012, 28:1086-1092.

90. Li R, Zhu H, Ruan J, Qian W, Fang X, Shi Z, Li Y, Li S, Shan G, Kristiansen K, Li S, Yang H, Wang J, Wang J: De novo assembly of human genomes with massively parallel short read sequencing. Genome Res 2010, 20:265-272.

91. Korf I: Gene finding in novel genomes. BMC Bioinformatics 2004, 5:59.
92. Stanke M, Tzvetkova A, Morgenstern B: AUGUSTUS at EGASP: using EST, protein and genomic alignments for improved gene prediction in the human genome. Genome Biol 2006, 7.

93. Gasser RB, Hu M, Chilton NB, Campbell BE, Jex AJ, Otranto D, Cafarchia C, Beveridge I, Zhu X: Single-strand conformation polymorphism (SSCP) for the analysis of genetic variation. Nat Protoc 2006, 1:3121-3128.

94. Li R, Fan W, Tian G, Zhu H, He L, Cai J, Huang Q, Cai Q, Li B, Bai Y, Zhang Z, Zhang Y, Wang W, Li J, Wei F, Li H, Jian M, Li J, Zhang Z, Nielsen R, Li D, Gu W, Yang Z, Xuan Z, Ryder OA, Leung FC, Zhou Y, Cao J, Sun X, Fu Y, et al: The sequence and de novo assembly of the giant panda genome. Nature 2010, 463:311-317.

95. Cock PJ, Fields CJ, Goto N, Heuer ML, Rice PM: The Sanger FASTQ file format for sequences with quality scores, and the Solexa/Illumina FASTQ variants. Nucleic Acids Res 2010, 38:1767-1771.

96. Kumar S, Blaxter ML: Simultaneous genome sequencing of symbionts and their hosts. Symbiosis 2011, 55:119-126.

97. Langmead B, Trapnell C, Pop M, Salzberg SL: Ultrafast and memoryefficient alignment of short DNA sequences to the human genome. Genome Biol 2009, 10:R25.

98. Langmead B, Salzberg SL: Fast gapped-read alignment with Bowtie 2. Nat Methods 2012, 9:357-359.

99. Zhang Z, Schwartz S, Wagner L, Miller W: A greedy algorithm for aligning DNA sequences. J Comput Biol 2000, 7:203-214.

100. Hess M, Sczyrba A, Egan R, Kim TW, Chokhawala H, Schroth G, Luo S, Clark DS, Chen F, Zhang T, Mackie RI, Pennacchio LA, Tringe SG, Visel A, Woyke T, Wang Z, Rubin EM: Metagenomic discovery of biomassdegrading genes and genomes from cow rumen. Science 2011, 6016:463-467.

101. Chilton NB, Huby-Chilton F, Gasser RB, Beveridge I: The evolutionary origins of nematodes within the order Strongylida are related to predilection sites within hosts. Mol Phylogenet Evol 2006, 40:118-128.

102. Zerbino DR, Birney E: Velvet: Algorithms for de novo short read assembly using de Bruijn graphs. Genome Res 2008, 18:821-829.

103. Hardie DC, Gregory TR, Hebert PD: From pixels to picograms: a beginners' guide to genome quantification by Feulgen image analysis densitometry. J Histochem Cytochem 2002, 50:735-749.

104. Parra G, Bradnam K, Ning Z, Keane T, Korf I: Assessing the gene space in draft genomes. Nucleic Acids Res 2009, 37:289-297.

105. Smit AFA, Robert $H$, Kas A, Siegel A, Gish W, Price A, Pevzner P: RepeatModeler. Institute of Systems Biology i, 1.0.5 2011.

106. Bao Z, Eddy SR: Automated de novo identification of repeat sequence families in sequenced genomes. Genome Res 2002, 12:1269-1276.

107. Price $A L$, Jones NC, Pevzner PA: De novo identification of repeat families in large genomes. Bioinformatics 2005, 21(Suppl 1):i351-358.

108. RepeatMasker Open-3.0. [http://www.repeatmasker.org].

109. Jurka J, Kapitonov W, Pavlicek A, Klonowski P, Kohany O, Walichiewicz J: Repbase Update, a database of eukaryotic repetitive elements. Cytogenet Genome Res 2005, 110:462-467.

110. Kent WJ: BLAT - The BLAST-like alignment tool. Genome Res 2002, 12:656-664.

111. Holt C, Yandell M: MAKER2: an annotation pipeline and genomedatabase management tool for second-generation genome projects. BMC Bioinformatics 2011, 12:491.

112. Altschul SF, Gish W, Miller W, Myers EW, Lipman DJ: Basic local alignment search tool. J Mol Biol 1990, 215:403-410.

113. Eilbeck K, Moore B, Holt C, Yandell M: Quantitative measures for the management and comparison of annotated genomes. BMC Bioinformatics 2009, 10:67.

114. Zdobnov EM, Apweiler R: InterProScan - an integration platform for the signature-recognition methods in InterPro. Bioinformatics 2001, 17:847-848.

115. Quevillon E, Silventoinen V, Pillai S, Harte N, Mulder N, Apweiler R, Lopez R: InterProScan: protein domains identifier. Nucleic Acids Res 2005, 33: W116-120.

116. Harris TW, Antoschechkin I, Bieri T, Blasair D, Chan J, Chen WJ, De La Cruz N, Davis P, Duesbury M, Fang R, Fernandes J, Han M, Kishore R, Lee $R$, Müller HM, Nakamura C, Ozersky P, Percherski A, Rangarajan A, Rogers A, Schindelman G, Schwarz EM, Tuli MA, Van Auken K, Wang D, Wang X, Williams G, Yook K, Durbin R, Stein LD, et al: WormBase: a comprehensive resource for nematode research. Nucleic Acids Res 2010, 38:D463-467. 
117. Magrane M, the UniProt Consortium: UniProt Knowledgebase: a hub of integrated protein data. Database (Oxford) 2011, 2011:bar009.

118. Gaulton A, Bellis LJ, Bento AP, Chambers J, Davies M, Hersey A, Light Y, McGlinchey S, Michalovich D, Al-Lazikani B, Overington JP: ChEMBL: a large-scale bioactivity database for drug discovery. Nucleic Acids Res 2012, 40:D1100-1107.

119. Plowman GD, Sudarsanam S, Bingham J, Whyte D, Hunter T: The protein kinases of Caenorhabditis elegans: a model for signal transduction in multicellular organisms. Proc Natl Acad Sci USA 1999, 96:13603-13610,

120. Manning G: Genomic overview of protein kinases. WormBook 2005, 1-19.

121. Saier MH Jr, Yen MR, Noto K, Tamang DG, Elkan C: The Transporter Classification Database: recent advances. Nucleic Acids Res 2009, 37: D274-278.

122. Saier MH Jr, Tran CV, Barabote RD: TCDB: the Transporter Classification Database for membrane transport protein analyses and information. Nucleic Acids Res 2006, 34:D181-186.

123. Kanehisa M, Goto S: KEGG: kyoto encyclopedia of genes and genomes. Nucleic Acids Res 2000, 28:27-30

124. Kanehisa M, Goto S, Sato Y, Furumichi M, Tanabe M: KEGG for integration and interpretation of large-scale molecular datasets. Nucleic Acids Res 2012, 40:D109-D114

125. Donizelli M, Djite MA, Le Novere N: LGICdb: a manually curated sequence database after the genomes. Nucleic Acids Res 2006, 34:D267-269.

126. Bellis LJ, Akhtar R, Al-Lazikani B, Atkinson F, Bento AP, Chambers J, Davies M, Gaulton A, Hersey A, Ikeda K, Krüger FA, Light Y, McGlinchey S, Santos R, Stauch B, Overington JP: Collation and data-mining of literature bioactivity data for drug discovery. Biochem Soc Trans 2011, 39:1365-1370.

127. NCBI Resource, Coordinators N: Database resources of the National Center for Biotechnology Information. Nucleic Acids Res 2013, 41:D8-D20.

128. Petersen TN, Brunak S, von Heijne G, Nielsen H: SignalP 4.0: discriminating signal peptides from transmembrane regions. Nat Methods 2011, 8:785-786.

129. Sonnhammer EL, von Heijne G, Krogh A: A hidden Markov model for predicting transmembrane helices in protein sequences. Proc Int Conf Intell Syst Mol Biol 1998, 6:175-182.

130. Krogh A, Larsson B, von Heijne G, Sonnhammer EL: Predicting transmembrane protein topology with a hidden Markov model: application to complete genomes. J Mol Biol 2001, 305:567-580.

131. Chen YJ, Zhang Y, Yin YB, Gao G, Li SG, Jiang Y, Gu XC, Luo JC: SPD - a web-based secreted protein database. Nucleic Acids Res 2005, 33: D169-D173.

132. Robinson MD, McCarthy DJ, Smyth GK: edgeR: a Bioconductor package for differential expression analysis of digital gene expression data. Bioinformatics 2010, 26(1):139-140.

133. R Development CoreTeam R: R: A language and environment for statistical computing. R Foundation for Statistical Computing. 2.15 edition. Vienna, Austria; 2011

134. Lohse M, Bolger AM, Nagel A, Fernie AR, Lunn JE, Stitt M, Usadel B: RobiNA: a user-friendly, integrated software solution for RNA-Seq-based transcriptomics. Nucleic Acids Res 2012, 40:W622-627.

135. Li H, Durbin R: Fast and accurate long-read alignment with BurrowsWheeler transform. Bioinformatics 2010, 26:589-595.

136. Li H, Handsaker B, Wysoker A, Fennell T, Ruan J, Homer N, Marth G, Abecasis G, Durbin R: The Sequence Alignment/Map format and SAMtools. Bioinformatics 2009, 25:2078-2079.

\section{Submit your next manuscript to BioMed Central and take full advantage of:}

- Convenient online submission

- Thorough peer review

- No space constraints or color figure charges

- Immediate publication on acceptance

- Inclusion in PubMed, CAS, Scopus and Google Scholar

- Research which is freely available for redistribution

Submit your manuscript at www.biomedcentral.com/submit 